\title{
Double-barred galaxies
}

\section{A catalog of barred galaxies with stellar secondary bars and inner disks ${ }^{\star}$}

\author{
Peter Erwin ${ }^{\star \star}$ \\ Instituto de Astrofísica de Canarias, C/ Via Láctea s/n, 38200 La Laguna, Tenerife, Spain
}

Received 27 September 2003 / Accepted 7 November 2003

\begin{abstract}
I present a catalog of 67 barred galaxies which contain distinct, elliptical stellar structures inside their bars. Fifty of these are double-barred galaxies: a small-scale, inner or secondary bar is embedded within a large-scale, outer or primary bar. I provide homogenized measurements of the sizes, ellipticities, and orientations of both inner and outer bars, along with global parameters for the galaxies. The other 17 are classified as inner-disk galaxies, where a large-scale bar harbors an inner elliptical structure which is aligned with the galaxy's outer disk. Four of the double-barred galaxies also possess inner disks, located in between the inner and outer bars. While the inner-disk classification is ad-hoc - and undoubtedly includes some inner bars with chance alignments (five such probable cases are identified) - there is good evidence that inner disks form a statistically distinct population, and that at least some are indeed disks rather than bars. In addition, I list 36 galaxies which may be double-barred, but for which current observations are ambiguous or incomplete, and another 23 galaxies which have been previously suggested as potentially being double-barred, but which are probably not. False double-bar identifications are usually due to features such as nuclear rings and spirals being misclassified as bars; I provide some illustrated examples of how this can happen.

A detailed statistical analysis of the general population of double-bar and inner-disk galaxies, as represented by this catalog, will be presented in a companion paper.
\end{abstract}

Key words. galaxies: structure - galaxies: elliptical and lenticular, $\mathrm{cD}$ - galaxies: spiral - galaxies: kinematics and dynamics

\section{Introduction}

The first hints that disk galaxies could have more than one bar emerged in the 1970s with observations by de Vaucouleurs $(1974,1975)$, who identified three galaxies where the largescale (outer or primary) bar harbored a concentric, smaller bar (the inner or secondary bar) in the nuclear region. Subsequent identifications of double-barred galaxies include those of Sandage \& Brucato (1979), Kormendy (1979, 1982a), and Schweizer (1980). Such bar-within-bar systems were generally thought to be isolated peculiarities, and there was essentially no theoretical interest in the topic.

Interest picked up in the late 1980s and early 1990s, spurred in part by a new theoretical angle: the idea that nested-bar systems might help fuel nuclear activity by efficiently driving gas into the nuclear regions of a galaxy, or even assist in building bulges out of disk material (Shlosman et al. 1989; Pfenniger $\&$ Norman 1990). The use of CCDs and near-infrared imagers allowed the detection of previously unnoticed double bars, and they began to be considered a distinct class of galaxies worthy of investigation and modeling (e.g., Buta \& Crocker 1993; Friedli \& Martinet 1993; Combes 1994). The latest studies,

* Tables 3 and 4 are only available in electronic form at http://www . edpsciences.org

$\star \star$ e-mail: erwin@ll.iac.es using well-defined samples and high-resolution imaging, suggest that as many as $\sim 1 / 3$ of all early-type barred galaxies may harbor secondary bars (Erwin \& Sparke 2002; Laine et al. 2002). There are even galaxies which some authors have identified as triple-barred (Wozniak et al. 1995; Erwin \& Sparke 1999; Laine et al. 2002), though at least some of these candidates have turned out, on closer inspection, to be only singleor double-barred (Erwin \& Sparke 1999; see Sects. 6 and 7 of this paper).

Inner bars are seen in both the optical (e.g., de Vaucouleurs 1975; Jarvis et al. 1988; Wozniak et al. 1995; Erwin \& Sparke 2003) and the near-infrared (e.g., Shaw et al. 1993, 1995; Friedli et al. 1996; Mulchaey et al. 1997; Jungwiert et al. 1997; Greusard et al. 2000; Laine et al. 2002). This latter fact, as well as their presence in S0 galaxies devoid of gas and dust, indicates that they are stellar structures, and thus at least broadly similar to "normal", large-scale bars. (Inner gaseous bars are sometimes seen as well, but these are not the subject of this catalog.)

Theoretical interest now includes hydrodynamical simulations of both observed galaxies (e.g., Knapen et al. 1995b; Ann 2001; Schinnerer et al. 2001) and model double-bar systems (Maciejewski et al. 2002; Shlosman \& Heller 2002). Questions concerning the formation, dynamical stability, and evolution of double bars have seen increasing attention from theorists (e.g., 
Friedli \& Martinet 1993; Friedli et al. 1996; Davies \& Hunter 1997; Maciejewski \& Sparke 1997; Rautiainen \& Salo 1999; Maciejewski \& Sparke 2000; Rautiainen et al. 2002; El-Zant \& Shlosman 2003). An intriguing result from the simulations of Rautiainen and collaborators is the suggestion that the inner bars of double-barred systems might form first, in contrast to the original outside-in formation scenario of Shlosman et al. (1989). If this is so, then inner bars would be among the oldest dynamical structures in these galaxies, and might provide useful clues about their formation and early history. There is also growing interest in spectroscopic studies specifically aimed at the kinematics of double-barred galaxies. Examples include long-slit spectroscopy by Emsellem et al. (2001), CO mapping by Schinnerer et al. (2001) and Petitpas \& Wilson (2002, 2003), and the 2-D optical spectroscopy of Moiseev and collaborators (Moiseev 2002; Moiseev et al. 2002).

Thus the time seems right for a first attempt at a comprehensive catalog of double-barred galaxies. Moiseev (2001) recently provided just such a list; however, there are several ways in which it can be improved. The main one is that Moiseev's list is fundamentally one of candidate double bars, with no attempt at confirmation or discrimination among alternate possible identifications. This means that some of the galaxies in the list are not, in fact, double-barred (as indeed Moiseev 2002 concluded on the basis of 2D spectroscopy). A number of suggested double-bar systems in the literature - including some of the recent spectroscopic targets - are either ambiguous or not truly double-barred; so there is also a need for identifying galaxies which can, under some circumstances, masquerade as double-barred. Finally, we would like to know more about the general population of double-barred galaxies: What Hubble types are they found in? How large and small can the inner (and outer) bars be, and what might this tell us about how they form and evolve? Can we identify differences between double- and single-barred galaxies?

This paper presents a catalog of confirmed double-bar and inner-disk galaxies, based on detailed examinations of over a hundred suggested candidates. For each galaxy, I provide measurements of bar sizes, orientations, and ellipticities in a consistent framework, along with basic data for the host galaxies. I also include a list of galaxies whose double-bar status is still ambiguous or unmeasurable, and a list of "false" double bars galaxies where nuclear rings, spiral arms, strong dust lanes, and the like have masqueraded as additional bars.

\section{Compilation and sources}

The starting point of this catalog was the survey of early-type barred galaxies carried out by Erwin \& Sparke (2002, 2003). That survey, however, found only ten double-barred galaxies, several of which had been identified by earlier studies, and an additional eight barred galaxies with inner disks ${ }^{1}$. To expand this to a statistically more meaningful collection, I have made a careful search for candidate double-barred galaxies in the literature, including previous compilations. Among the surveys and compilations examined were those of Kormendy (1979);

\footnotetext{
${ }^{1}$ See Sect. 3.2 for the definition of inner disks.
}

Shaw et al. (1993, 1995); Buta \& Crocker (1993); Friedli \& Martinet (1993); Wozniak et al. (1995); Friedli et al. (1996); Friedli (1996); Mulchaey et al. (1997); Jungwiert et al. (1997); Márquez et al. (1999); Greusard et al. (2000); Martini et al. (2001); Rest et al. (2001); Moiseev (2001); and Laine et al. (2002). Finally, a number of unpublished observations, both ground-based and from the HST archive, were examined; this turned up several more double-barred galaxies. (These observations will be presented in Erwin et al. 2004, in prep.) The goal has been to find as many plausible candidates as possible, though it is probably guaranteed that I have missed some previously reported candidates along the way.

Because the sources are so many and varied, and motivated by a variety of different aims, this catalog does not represent a uniform or complete sample. For a variety of reasons, it is biased towards early-type and active galaxies, and generally consists of galaxies with larger and stronger inner bars than may be the norm, since these are the ones most likely to be noticed and mentioned in the literature. It is probably not the best sample for probing, for example, the lower limits of inner-bar sizes; on the other hand, it probably is a good resource for investigating the upper range of their sizes, a question of some theoretical interest (Maciejewski \& Sparke 2000; El-Zant \& Shlosman 2003).

\subsection{Evaluation and winnowing}

With the candidates in hand $(\sim 125$ galaxies in all $)$, the next step was to determine which galaxies were actually double-barred: as I will discuss below, there are a number of features which can masquerade as inner (or outer) bars, particularly if ellipse fitting is the main or only tool used to identify bars. Because one of the main goals of this catalog was to produce a set of homogeneous bar measurements, I also needed galaxies where the bar characteristics had in fact been measured, or for which publically available images existed. This winnowing process produced three sorts of chaff: galaxies which could be doublebarred, but for which no measurements were available or could be made; galaxies for which the evidence for a double bar is ambiguous (often cases where dust or lack of resolution confuses the issue); and galaxies for which the evidence argues against a double bar, usually because rings, spiral arms, and/or strong dust lanes have produced false bar signatures. These unconfirmed, "ambiguous" and "discarded" galaxies are listed in Sects. 6 and 7, along with the reasons for each classification.

How does one verify the existence of an inner bar, or determine that an apparent inner bar is really something else? To start with, Fig. 1 gives an example of a prototypical doublebar galaxy. Both inner and outer bars are bisymmetric, approximately elliptical stellar structures. By definition, an inner or secondary bar must appear inside a larger bar - so part of the verification process is ensuring that there is indeed a large-scale bar in the galaxy. Bars usually appear with a characterisic distortion in the isophotes (weaker for inner bars, in part because light from the bulge makes the isophotes rounder than they would otherwise be), usually producing a peak in the isophotal ellipticity at the approximate position angle (PA) of the inner 


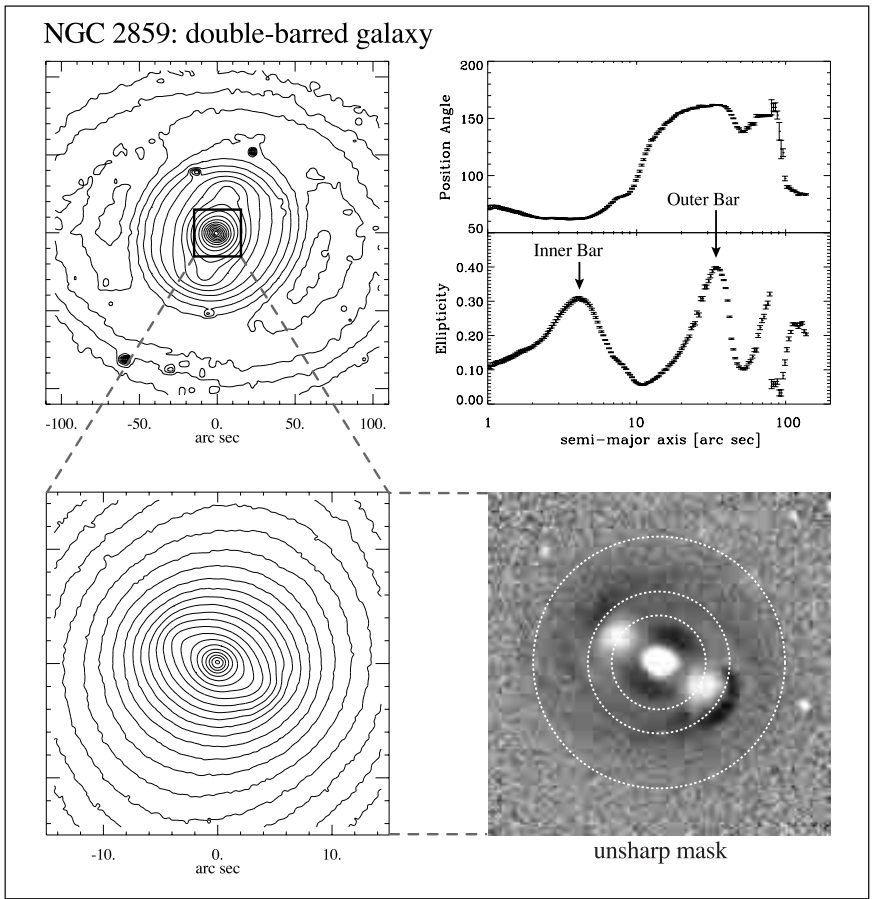

Fig. 1. NGC 2859, a prototypical double-barred galaxy first noted by Kormendy (1979). The figure shows logarithmic $R$-band contours, displaying both the outer bar and the inner bar inside it, along with ellipse fits to the isophotes (upper right) and an unsharp mask (lower right) of the inner bar, with hints of spiral arms just outside it. The dashed circles on the unsharp mask have, from the inside out, radii $=$ $a_{\epsilon}, a_{10}$, and $a_{\min }$, three different ellipse-fit-based measures of bar size; see Sect. 3.1 for definitions. (See Erwin \& Sparke 2003 for more details on the observations.)

bar. A common technique for finding bars, therefore, is to fit ellipses to the isophotes, and look for peaks in ellipticity accompanied by relatively stationary position angles (e.g., Wozniak et al. 1995; Laine et al. 2002). The problem, however, is that there are other structures which can produce similar distortions and similar features in ellipse fits, as I will show below (see also Erwin \& Sparke 2003). These include nuclear rings, nuclear spirals, strong dust lanes, and star formation. Thus, it is necessary to go further: to inspect the images or isophotes directly, and to make use of tools such as unsharp masking, which can help discriminate between these different structures.

As an example, Fig. 2 shows how large-scale bars, including the outer bars of double-barred galaxies, appear in unsharp masks. Depending on how narrow and strong the bar is, the unsharp mask can show the ridgeline of the bar (e.g., NGC 4643 in the figure), or just the characteristic sharp ends typical of so-called "flat" or "early-type" bars, where the bar's majoraxis luminosity profile steepens abruptly (Kormendy 1982b; Elmegreen \& Elmegreen 1985). What Fig. 3 shows is that inner bars have a very similar appearance to large-scale bars in unsharp masks. This is an indication that inner and outer bars are structurally similar (Erwin 2004b, hereafter Paper II), and it also suggests that unsharp masking can be used as a secondary technique for identifying and confirming the presence of inner bars.

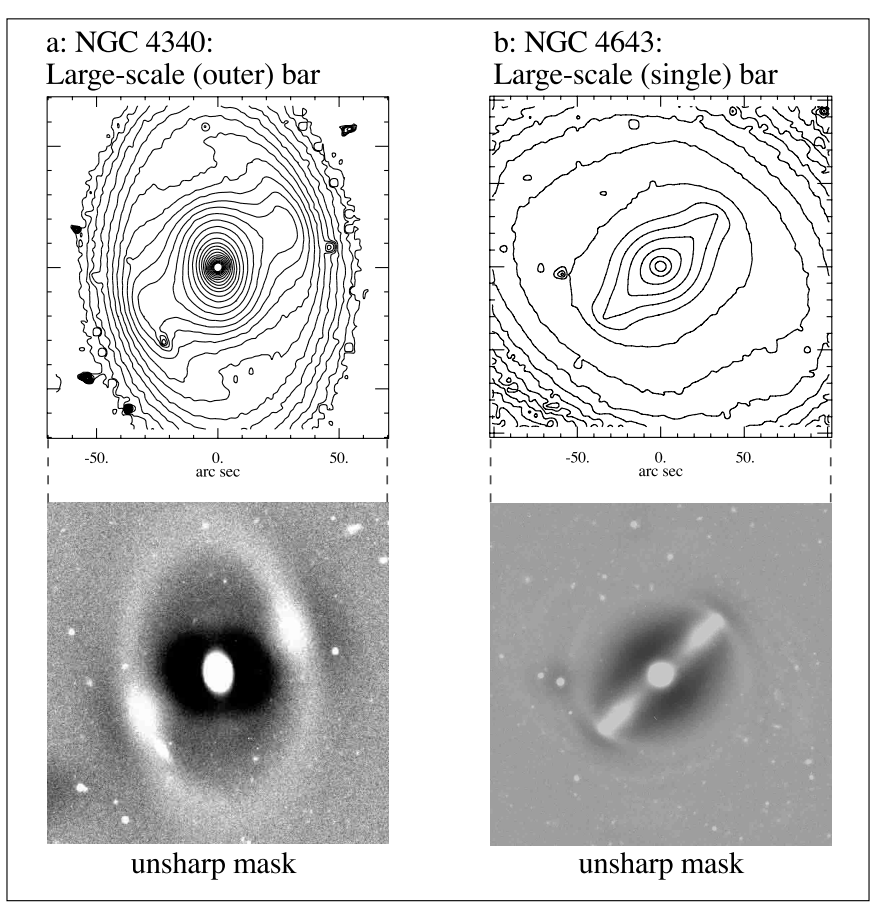

Fig. 2. Examples of the appearance of "normal" (i.e., large-scale) bars in unsharp masks. For each galaxy I show logarithmically scaled isophotes (top) and an unsharp mask from the same image (bottom). a) The outer bar of the double-barred SB0 galaxy NGC 4340; traces of a ring surrounding the bar can also be seen ( $R$-band image from the MDM Telescope). b) NGC 4643, an example of an extremely narrow bar in an SB0/a galaxy, with the central "spine" of the bar showing up clearly in the unsharp mask, along with very faint traces of a thin ring ( $R$-band image from Erwin \& Sparke 2003).

Two examples of how nuclear rings can masquerade as inner bars are shown in Figs. 4 and 5. In both cases, there is a clear peak in the ellipticity, with a stationary or extremal value of the position angle, inside the large-scale ("outer") bar; these features are seen in near-infrared or dust-free $R$-band images, and so are unlikely to be due to dust lanes (though it must be kept in mind that sufficient dust can affect even $K$-band images). So on purely ellipse-fit criteria these are multiply barred galaxies. However, unsharp masking shows that the inner elliptical features are clearly rings, with no evidence for barlike structures inside the rings.

Sometimes the inner bar is real, but the outer bar is not: Fig. 6 demonstrates how spiral arms can produce a false bar signature in the ellipse fits. In other cases, the presence of an outer bar may be uncertain, usually due to confusion from dust and spiral arms, or because the putative outer bar is so round or peculiarly shaped that it isn't clear whether it is a bar or not; this can include structures sometimes called "oval disks" (e.g. Kormendy \& Norman 1979). In two such oval-disk galaxies (NGC 1068 and NGC 4736), there is both good evidence for small-scale bars and sufficient kinematic evidence that the oval disks are dynamically barlike, so I consider these to be doublebarred galaxies (albeit with rather weak, atypical outer bars). 


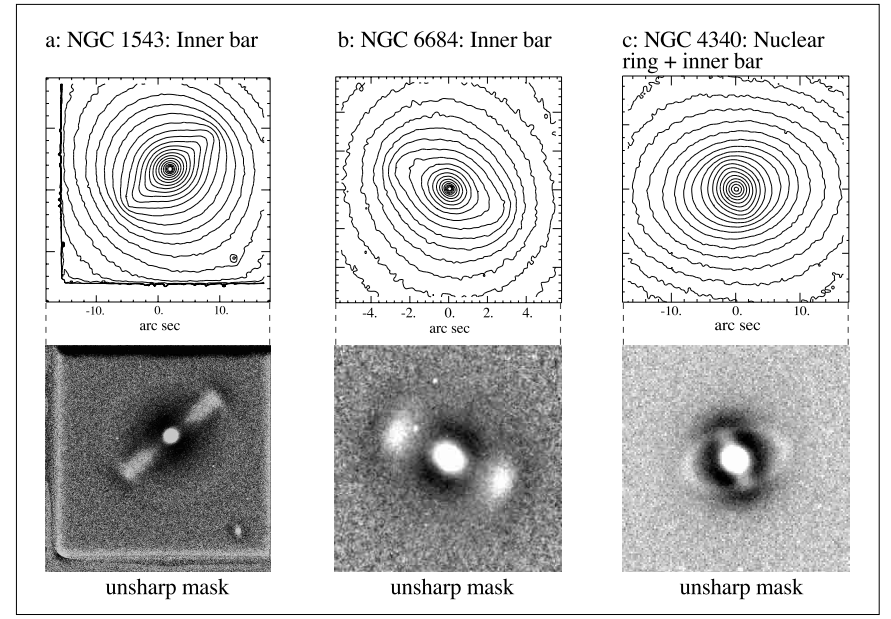

Fig. 3. As for Fig. 2, but now showing inner bars from double-barred galaxies. a) NGC 1543, where the narrow inner bar is strikingly similar to the (large-scale) bar of NGC 4643 in Fig. 2 (WFPC2 F814W image). b) NGC 6684, an inner bar with a more typical unsharp mask appearance (WFPC2 F814W image). c) NGC 4340, where the inner bar is surrounded by an elliptical stellar nuclear ring ( $R$-band image from the MDM Telescope).

\section{Definitions and measurements}

\subsection{How to measure bars}

The three basic measurements one can make of a bar (or inner disk) are its strength, its size, and its orientation. The latter is, in principle, the simplest and least ambiguous measurement, and is defined as the position angle (PA), measured east from north (though there are some caveats about how to measure it; see below). The other two measurements are more difficult, both to define and to make. Bar "strength" is probably the most ambiguous and contentious. As Athanassoula (2003) notes, "Although the notion of bar strength is clear to everyone, and it is very often easy, when comparing two bars, to say which one is strongest, a precise definition is not trivial". I have defaulted to using one of the simplest definitions - the maximum ellipticity $\left(\epsilon_{\max }\right)$ of the isophotes in the bar region - because it is used rather often for bars (e.g., Martin 1995; Wozniak et al. 1995; Jungwiert et al. 1997; Laine et al. 2002; Erwin \& Sparke 2003) and because it is easy to determine from published ellipse fits; this lets me determine bar strengths for as many of the galaxies as possible. Laurikainen et al. (2002) have recently shown that bar $\epsilon_{\max }$ correlates quite well with the more sophisticated (and difficult) measurement based on maximum relative tangential force due to the bar (Buta \& Block 2001).

For measuring the lengths of bars, I adopted a stratagem similar to those of Erwin \& Sparke (2003) and Erwin (2004a), which is to define two radial sizes: $a_{\epsilon}$ and $L_{\text {bar }}$. From the ellipse fits, I measured the semi-major axis at maximum ellipticity $\left(a_{\epsilon}\right)$; the semi-major axis at the first minimum in ellipticity outside the bar $\left(a_{\min }\right.$; based on the approach of Wozniak et al. 1995); and the semi-major axis $a_{10}$ where the PA has changed by $10^{\circ}$ from the bar PA. All such values, when they can be measured, are listed in Table 3 . The maximum-ellipticity length $a_{\epsilon}$ is adopted as one measure of the bar size, in part

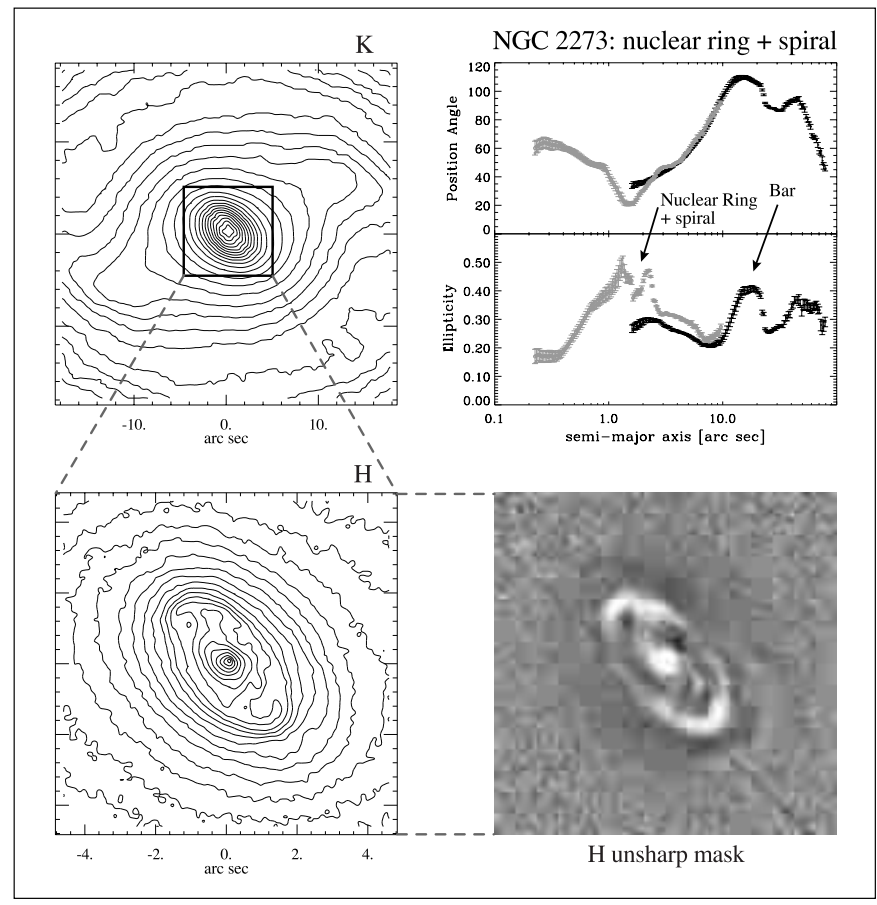

Fig. 4. Example of a single-barred galaxy where a nuclear ring masquerades as an inner bar. The SBa galaxy NGC 2273 was listed as double-barred by Mulchaey et al. (1997) on the basis of ellipse fits (upper right: black $=K$-band fits to their image, gray $=H S T H$-band fits) and the appearance of their $K$-band image (upper left). However, an $H S T$ NICMOS2 $H$-band image (lower left) and its unsharp mask (lower right) show that the "inner bar" $\left(a \sim 2^{\prime \prime}\right)$ is actually a nuclear ring, with a nuclear spiral inside; WFPC2 images and color maps confirm this (see Erwin \& Sparke 2003; Martini et al. 2003).

because it is a relatively simple and repeatable measurement and is used rather often, including for some $n$-body bars (e.g., Wozniak \& Pierce 1991; Jungwiert et al. 1997; Laine et al. 2002; Athanassoula \& Misiriotis 2002). It generally indicates the radius at which the isophotal distortion due to the bar is at a maximum; this is often close to the radius where the bar's major-axis profile turns over, at least in early-type galaxies.

In some cases, there is no clearly defined maximum ellipticity for the bar. Usually, however, there is a local maximum or minimum in the position angle, which again represents the point where the bar has its strongest effect on the isophotes. This semi-major axis then becomes $a_{\epsilon}$. (See Nieto et al. 1992; Busarello et al. 1996; Erwin \& Sparke 2003 for examples.)

Despite the common use of $a_{\epsilon}$, there is good evidence that it is usually an underestimate of the bar's true length (e.g., Wozniak et al. 1995; Laurikainen et al. 2002; Athanassoula \& Misiriotis 2002; Erwin \& Sparke 2003); see Fig. 1. So Erwin $\&$ Sparke defined a second measurement, which I refer to here as $L_{\mathrm{bar}}$, by taking the smaller of $a_{\min }$ and $a_{10}$. In certain cases, I use an alternate measurement to define $L_{\mathrm{bar}}$, such as the size of an inner or nuclear ring surrounding the bar, when it is clear that the bar does not extend past the ring and that $a_{\min }$ and $a_{10}$ are well outside the bar or simply cannot be defined (these cases are noted in Sect. 5); the presence of such a ring can distort the ellipse fits and produce misleadingly large values of $a_{\text {min }}$ and $a_{10}$. 


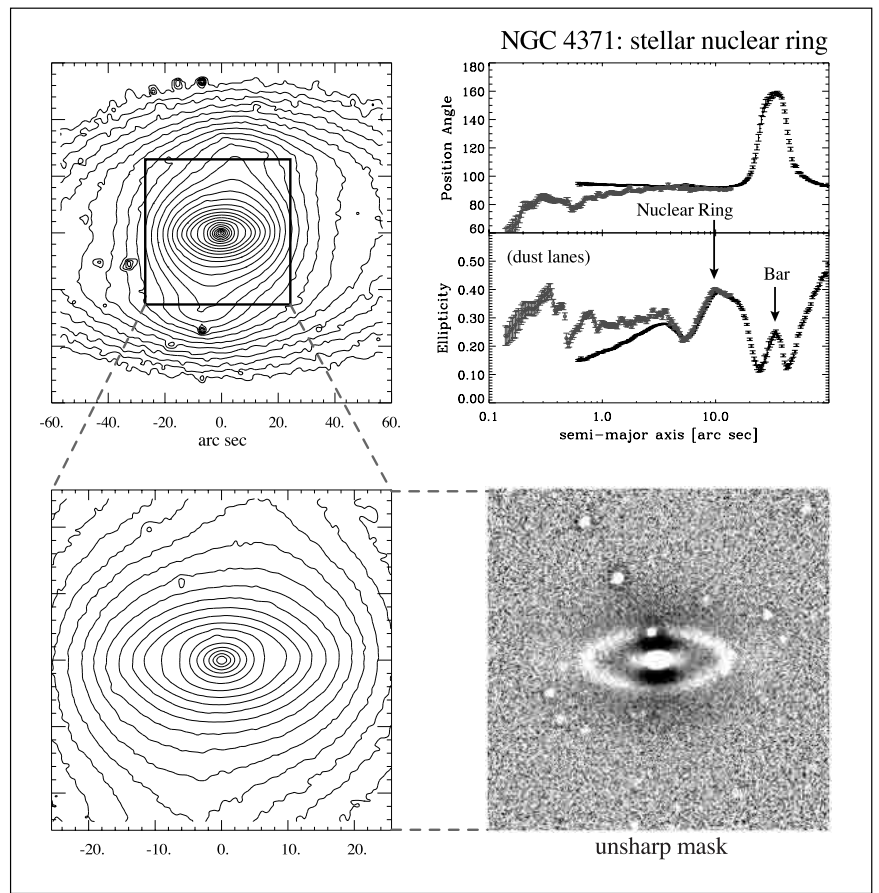

Fig. 5. Another example of a nuclear ring masquerading as an inner bar. Here, the SB0 galaxy NGC 4371 shows three distinct peaks in ellipse fits to ground-based images (upper right: black = ground-based $R$-band fits, gray $=$ WFPC2 $V$-band fits; both from Erwin \& Sparke 1999), which led Wozniak et al. (1995) to suggest that it was triplebarred. The $R$-band images (upper and lower left) do indeed show a prominent inner elliptical feature with $a \sim 10^{\prime \prime}$, corresponding to the middle ellipticity peak; but unsharp masking (bottom right) shows that this is due to a stellar nuclear ring. The innermost ellipticity peak (in the ground-based ellipse fits) is actually due to the combination of seeing effects and the superposition of an elliptical ring on top of rounder bulge isophotes (see Erwin \& Sparke 1999; Erwin et al. 2001). Circumnuclear dust lanes produce variable ellipticity and position angles in the HST fits at $a<1^{\prime \prime}$.

In Erwin (2004a), I find that $a_{\epsilon}$ and $L_{\text {bar }}$, as defined above, correlated extremely well for the primary (or single) bars of a sample of S0-Sb galaxies. The same is true here: the Pearson and Spearman correlation coefficients are $r=0.93$ and $r_{\mathrm{s}}=0.95$ for inner and outer bars, respectively. The mean ratio of $a_{\epsilon} / L_{\mathrm{bar}}$ is $0.75 \pm 0.12$ for the inner bars and $0.79 \pm 0.12$ for the outer bars. This is almost identical to the ratio for $\mathrm{S} 0-\mathrm{Sb}$ primary or single bars $(0.80)$ and similar to the mean ratio of Athanassoula \& Misiriotis' (2002) $L(\mathrm{a} / \mathrm{b})$ and $L$ (phase) bar-size measurements (0.73), applied to bars in $n$-body simulations.

Most of the bar measurements are based, directly or indirectly, on fitting ellipses to the isophotes. The actual ellipse-fit values come from three sources: ellipse fits performed by myself on available images; examination of published ellipse fits; and reported values when the actual fits were not published. Sources for the latter two are given for each galaxy in Sect. 5.

Although bar position angle is, as noted above, generally the simplest and least ambiguous measurement, there can be problems if ellipse fits are being used for bar measurements. Ellipse-fit position angles have the advantage of being (usually) precise and repeatable; however, it must be remembered

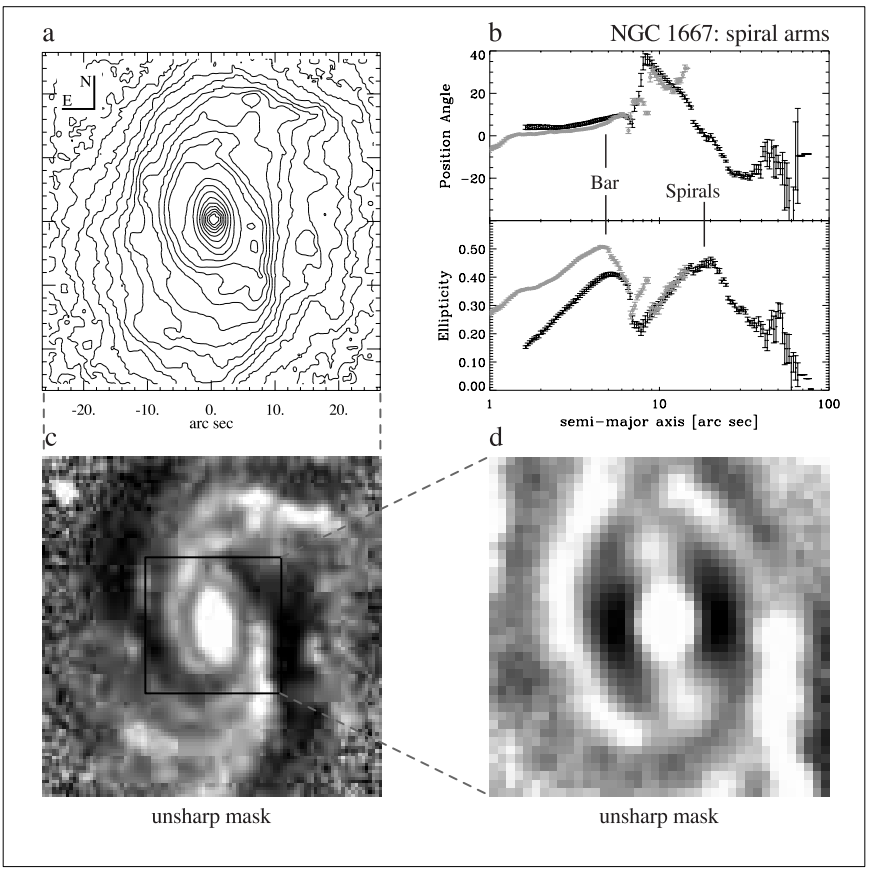

Fig. 6. Another example of how ellipse fits can misleadingly suggest two bars in a single-barred galaxy: in this case, a false "outer bar" signature is produced by spiral arms in the SABc galaxy NGC 1667. a) Logarithmically spaced $K$-band isophotes (from the image of Mulchaey et al. 1997), along with ellipse fits b) to the same image (black points) plus ellipse fits to the NICMOS2 $\mathrm{H}$-band image (gray points). Two strong ellipticity peaks are present, suggesting a possible double-barred galaxy (e.g., Laine et al. 2002): an "outer bar" with $a \sim 20$ " and an "inner bar" with $a \sim 5^{\prime \prime}$. However, unsharp masking c) with Gaussian $\sigma=10$ pixels shows that the "outer bar" feature is really due to spiral arms (Mulchaey et al.). In contrast, unsharp masking with $\sigma=3$ pixels d) shows that the "inner bar" is indeed a baralmost certainly the galaxy's only bar.

that they are fits to isophotes, and are sometimes a compromise. Multiple components (bar + bulge, or bar + spirals) and projection effects can conspire to produce isophotes for which the best-fit ellipse has a position angle which does not match any of the individual components. As discussed and illustrated by Erwin $\&$ Sparke (2003), there are numerous cases where the position angle of the bar, based on its orientation in the image, does not match the position angle from the ellipse fits. They found that the ellipse-fit position angle for bars was off by $>5^{\circ}$ for about one-third of the large-scale (outer or single) bars in their sample. The problem is worse for inner bars, in part because their isophotes are usually contaminated by light from the inner bulge, nuclear rings, and inner disks: Erwin \& Sparke found that inner-bar position angles differed by $>10^{\circ}$ from the ellipse-fit position angles for about half of their doublebarred galaxies. Because of these problems, all bar position angles have been checked, wherever possible, by direct inspection of the isophotes and unsharp masks. If this indicated that the ellipse-fit PA value was off by more than $\sim 2-3^{\circ}$, then the ellipse-fit value was discarded and the bar PA was measured directly from the image.

A simple example: Consider the case of NGC 2859 (Fig. 1). The ellipse fits show two clear peaks in ellipticity, with 
$a_{\epsilon}=4.1^{\prime \prime}$ and $a_{\epsilon}=34^{\prime \prime}$ for the inner and outer bars, respectively (the peaks at larger radii are due to the lens and outer ring). The isophotes clearly indicate bars rather than spirals, and unsharp masking supports this. For the inner bar, there is an unambiguous minimum in ellipticity at $a_{\min }=11^{\prime \prime}$; but since the position angle has changed by $10^{\circ}$ from the bar PA $\left(62^{\circ}\right)$ by the time $a=6.2^{\prime \prime}$, I adopt the latter value $\left(a_{10}\right)$ as $L_{\text {bar. }}$. (There $i s$ a faint, dusty nuclear ring surrounding the inner bar - see Erwin $\&$ Sparke 2003 - but since it has $a=7^{\prime \prime}$, which is $>a_{10}$, I keep $L_{\mathrm{bar}}=a_{10}$.) For the outer bar, there is a clear ellipticity minimum at $a_{\mathrm{min}}=52^{\prime \prime}$, but again $a_{10}$ is smaller (43") than $a_{\mathrm{min}}$, so $L_{\text {bar }}$ is set $=a_{10}$.

\subsection{Inner disks}

Following Erwin \& Sparke (2002, 2003), I classify some of the inner elliptical structures as "inner disks". The criteria for this are: measured ellipticity less than that of the outer disk, measured position angle differing by $\leq 10^{\circ}$ from that of the outer disk, and no evidence that the structure is a nuclear ring or spiral (e.g., from unsharp masking or color maps). As pointed out by Erwin \& Sparke, this makes for a heterogeneous, hodgepodge of a category, which can include genuine disks, inner bars with chance alignments, unresolved stellar rings, and flattened bulges. Erwin \& Sparke (2002) were able to find statistical evidence that the inner disks constituted a distinct category, different from inner bars (primarily because they are larger relative to the outer bars); Paper II shows that this statistical distinction is even stronger when the much larger set of galaxies in this catalog is considered. There is also evidence that at least some of the inner disks really are disks: Erwin et al. (2003) showed that the inner disks of NGC 2787 and NGC 3945 are morphologically, photometrically, and kinematically disklike.

Nevertheless, some of the "inner disks" presented here probably are inner bars which happen to be aligned with the outer disk, particularly when the combination of innerbar and bulge light produces composite isophotes which are rounder than the inner bar would be by itself. (NGC 2642 and NGC 7098, on the other hand, are examples of double-barred galaxies where the inner bar is closely aligned with the outer disk, but is clearly more elliptical; thus, they are not classified as inner disks.) A few candidates can be identified on the basis of unsharp masking - that is, unsharp masking suggests a typical bar structure, rather than the smoother, elliptical appearance of most inner disks; compare Figs. 2 and 3 with Fig. 1 of Erwin et al. (2003). These galaxies are indicated by notes in Table 2.

\subsection{Deprojection}

To compare the sizes and orientations of bars and disks, I deproject their measurements using the outer-disk inclinations and positions angles from Cols. 5 and 6 of Tables 1 and 2, assuming that bars are relatively flat, linear structures. Recent near-IR studies of edge-on galaxies (Lütticke et al. 2000) show that large-scale bars are indeed flat over most of their length, with only the inner $\sim 30-40 \%$ of the bar being thick (in the form of peanut-shaped bulges). Whether inner bars would have their own vertical thickening is unknown, though the high pattern speeds suggested for inner bars would probably preclude vertical Lindblad resonances outside the very nucleus.

Galaxies with $i<25^{\circ}$ are not deprojected, both because deprojection has little effect for inclinations that small (lengths increase by $10 \%$ at the very most) and because such galaxies often do not have a well-defined major-axis position angle for the outer disk.

\section{The catalog}

The final catalog is presented in Tables 1 and 2, divided into double-barred galaxies and inner-disk galaxies. Measurements for the bars (outer and inner) and inner disks are given in Table 3, with deprojected bar sizes and relative position angles ( $\triangle \mathrm{PA}$ ) in Table 4. For $\triangle \mathrm{PA}$, I follow Buta \& Crocker (1993) and use a convention where the inner bar (or inner disk) "leads" or "trails" the outer bar, depending on the sense of rotation given by the spirals (Col. 8 of Tables 1 and 2); see Fig. 5 of Buta \& Crocker. Since NGC 2681 has three bars, the relative position angle is harder to define - e.g., which bar counts as the "outer bar" with respect to the innermost bar? - so there is no $\triangle \mathrm{PA}$ for that galaxy in Table 4. The galaxy is almost face-on $\left(i \approx 18^{\circ}\right)$, so the interested reader can determine the various possible relative position angles using the observed bar position angles in Table 3, without needing to do a deprojection.

Details and special notes for these galaxies are given in Sect. 5. This includes discussions of sources for identifications and measurements, how the outer-disk orientation was derived - e.g., from the outer isophotes, from H I kinematics, etc. - and other items of interest.

There are a number of other candidate double-barred or inner-disk galaxies which I do not list in the tables; these are discussed in Sect. 6. These are primarily cases where good measurements of one or both bars are not yet available, or where alternate explanations - strong dust lanes, nuclear rings, etc. - cannot be ruled out using the images currently available. The most promising candidate (i.e., galaxies which are probably double-barred, but for which good measurements are not available) are NGC 2442, NGC 4274, and IC 454. One particularly interesting, though admittedly ambiguous, case is the Milky Way itself, which Alard (2001) recently suggested might be double-barred.

Finally, candidate galaxies which were rejected as being single-barred (or even unbarred) are listed in Sect. 7, along with the reasons for rejection.

A statistical analysis of these measurements will be presented in Paper II.

\section{Notes on individual galaxies}

For each galaxy, I first list the "source" - i.e., the citation(s) where the double-bar identification was first made, along with significant early studies of the double-bar/inner-disk system as such - and then briefly discuss where the bar, disk, and other measurements come from. Unless otherwise noted, outer-disk position angle and inclination are taken from the 
Table 1. General data for double-barred galaxies.

\begin{tabular}{|c|c|c|c|c|c|c|c|c|c|}
\hline Name & Type (RC3) & $\overline{B_{\mathrm{t}, \mathrm{c}}}$ & $\begin{array}{r}R_{25} \\
\prime \prime \\
(4)\end{array}$ & $\begin{array}{r}i \\
\circ \\
(5)\end{array}$ & $\begin{array}{r}\text { Disk PA } \\
\circ \\
(6)\end{array}$ & $\begin{array}{r}\text { Distance } \\
\mathrm{Mpc} \\
(7)\end{array}$ & $\begin{array}{r}\sigma_{0} \\
\mathrm{~km} \mathrm{~s}^{-1} \\
(8)\end{array}$ & Rot & $\begin{array}{r}\text { Notes } \\
(10)\end{array}$ \\
\hline NGC 357 & $\mathrm{SB}(\mathrm{r}) 0 / \mathrm{a}$ & 12.49 & 72 & 37 & 20 & 31.6 & & - & \\
\hline NGC 718 & $\mathrm{SAB}(\mathrm{s}) \mathrm{a}$ & 12.34 & 71 & 30 & 5 & 22.6 & 127 & + & \\
\hline NGC 1068 & (R)SA(rs)b & 9.23 & 212 & 40 & 98 & 14.7 & 148 & + & \\
\hline NGC 1097 & $\left(\mathrm{R}_{1}^{\prime}\right) \mathrm{SB}(\mathrm{rl}) \mathrm{b}$ & 9.76 & 279 & 46 & 134 & 14.3 & $\ldots$ & - & \\
\hline NGC 1241 & $\mathrm{SB}(\mathrm{rs}) \mathrm{b}$ & 12.11 & 84 & 55 & 145 & 52.1 & $\ldots$ & + & \\
\hline NGC 1291 & $\left(\mathrm{R}_{1}^{\prime}\right) \mathrm{SB}(\mathrm{l}) 0 / \mathrm{a}$ & 9.49 & 293 & 6 & $\ldots$ & 7.9 & 186 & + & \\
\hline NGC 1317 & $\left(\mathrm{R}^{\prime}\right) \mathrm{SAB}(\mathrm{rl}) 0 / \mathrm{a}$ & 11.74 & 83 & 30 & 78 & 19.3 & $\ldots$ & - & \\
\hline NGC 1433 & $\left(\mathrm{R}_{1}^{\prime}\right) \mathrm{SB}(\mathrm{rs}) \mathrm{ab}$ & 10.54 & 194 & 33 & 21 & 10.8 & $\ldots$ & - & \\
\hline NGC 1543 & $(\mathrm{R}) \mathrm{SB}(1) 0^{0}$ & 11.39 & 147 & 20 & $\ldots$ & 20.0 & 158 & $\ldots$ & \\
\hline NGC 1808 & $\left(\mathrm{R}_{1}^{\prime}\right) \mathrm{SAB}(\mathrm{s}:) \mathrm{b}$ & 10.30 & 194 & 50 & 133 & 10.2 & 148 & + & \\
\hline NGC 2217 & (R) $\mathrm{SB}(\mathrm{rs}) 0^{+}$ & 11.36 & 134 & 22 & 5 & 18.9 & 232 & + & \\
\hline NGC 2642 & $\mathrm{SB}(\mathrm{r}) \mathrm{bc}$ & 13.07 & 61 & 24 & 140 & 56.8 & $\ldots$ & - & \\
\hline NGC 2646 & $\mathrm{SB}(\mathrm{r}) 0^{0}$ & 13.53 & 40 & 21 & $\ldots$ & 52.2 & 222 & $\ldots$ & \\
\hline NGC 2681 & $\left(\mathrm{R}^{\prime}\right) \mathrm{SAB}(\mathrm{rs}) 0 / \mathrm{a}$ & 10.98 & 109 & 18 & 140 & 17.2 & 111 & + & 1 \\
\hline NGC 2859 & $(\mathrm{R}) \mathrm{SB}(\mathrm{r}) 0^{+}$ & 11.71 & 128 & 25 & 90 & 24.3 & 177 & + & \\
\hline NGC 2950 & $(\mathrm{R}) \mathrm{SB}(\mathrm{r}) 0^{0}$ & 11.73 & 80 & 48 & 120 & 14.9 & 183 & + & \\
\hline NGC 2962 & $(\mathrm{R}) \mathrm{SAB}(\mathrm{rs}) 0^{+}$ & 12.72 & 79 & 53 & 10 & 30.0 & 192 & - & \\
\hline NGC 3081 & $\left(\mathrm{R}_{1}^{\prime}\right) \mathrm{SAB}(\mathrm{r}) 0 / \mathrm{a}$ & 12.71 & 63 & 34 & 97 & 29.9 & $\ldots$ & + & \\
\hline NGC 3275 & $\mathrm{SB}(\mathrm{r}) \mathrm{a}$ & 11.98 & 85 & 42 & 122 & 40.5 & $\ldots$ & + & \\
\hline NGC 3358 & $\left(\mathrm{R}_{2}^{\prime}\right) \mathrm{SAB}(1) \mathrm{ab}$ & 11.85 & 99 & 42 & 125 & 37.3 & $\ldots$ & - & \\
\hline NGC 3368 & $\mathrm{SAB}(\mathrm{rs}) \mathrm{ab}$ & 9.75 & 228 & 50 & 172 & 10.5 & 114 & - & 2 \\
\hline NGC 3393 & $\left(\mathrm{R}^{\prime}\right) \mathrm{SB}(\mathrm{s}) \mathrm{ab}$ & 12.79 & 66 & 25 & $\ldots$ & 48.3 & 184 & + & \\
\hline NGC 3941 & $\mathrm{SB}(\mathrm{s}) 0^{0}$ & 11.13 & 104 & 51 & 10 & 12.2 & 175 & $+?$ & \\
\hline NGC 3945 & (R) $\mathrm{SB}(\mathrm{rs}) 0^{+}$ & 11.54 & 158 & 50 & 158 & 19.3 & 165 & $-?$ & 2 \\
\hline NGC 4303 & $\mathrm{SAB}(\mathrm{rs}) \mathrm{bc}$ & 10.04 & 194 & 25 & 138 & 15.3 & 96 & - & \\
\hline NGC 4314 & $\mathrm{SB}(\mathrm{rs}) \mathrm{a}$ & 11.28 & 125 & 25 & 65 & 12.0 & 117 & + & \\
\hline NGC 4321 & $\mathrm{SAB}(\mathrm{s}) \mathrm{bc}$ & 9.79 & 222 & 27 & 153 & 15.2 & 94 & + & \\
\hline NGC 4340 & $\mathrm{SB}(\mathrm{r}) 0^{+}$ & 11.99 & 105 & 50 & 95 & 15.3 & 113 & - & \\
\hline NGC 4503 & $\mathrm{SB}^{-}$: & 11.82 & 106 & 64 & 12 & 15.3 & 118 & + & \\
\hline NGC 4725 & $\mathrm{SAB}(\mathrm{r}) \mathrm{ab}$ & 9.74 & 321 & 42 & 40 & 12.4 & 155 & - & \\
\hline NGC 4736 & (R)SA(r)ab & 8.68 & 337 & 35 & 113 & 5.2 & 136 & - & \\
\hline NGC 4785 & $\left(\mathrm{R}^{\prime}\right) \mathrm{SAB}(\mathrm{r}) \mathrm{ab}$ & 11.80 & 59 & 64 & 81 & 46.4 & 186 & - & 2 \\
\hline NGC 4984 & $(\mathrm{R}) \mathrm{SAB}(\mathrm{rs}) 0^{+}$ & 11.96 & 84 & 40 & 15 & 15.6 & $\ldots$ & $\ldots$ & \\
\hline NGC 5365 & (L) $\mathrm{SB}(\mathrm{s}) 0^{-}$ & 11.92 & 89 & 48 & 4 & 30.5 & 232 & + & \\
\hline NGC 5728 & $\left(\mathrm{R}_{1}^{\prime}\right) \mathrm{SAB}(\mathrm{r}) \mathrm{a}$ & 11.79 & 93 & 40 & 2 & 36.9 & 209 & - & \\
\hline NGC 5850 & $\mathrm{SB}(\mathrm{r}) \mathrm{b}$ & 11.45 & 128 & 30 & 163 & 35.2 & $\ldots$ & + & \\
\hline NGC 6654 & $\left(\mathrm{R}^{\prime}\right) \mathrm{SB}(\mathrm{s}) 0 / \mathrm{a}$ & 12.63 & 79 & 44 & 0 & 28.3 & $\ldots$ & - & \\
\hline NGC 6684 & (L)SB(r) $0^{+}$ & 11.00 & 119 & 51 & 36 & 13.9 & 110 & $\ldots$ & \\
\hline NGC 6782 & $\left(\mathrm{R}_{1}^{\prime}\right) \mathrm{SB}(\mathrm{r}) 0 / \mathrm{a}$ & 12.26 & 66 & 41 & 45 & 48.5 & 140 & + & \\
\hline NGC 7098 & (R)SAB(r)a & 11.94 & 122 & 42 & 75 & 28.0 & $\ldots$ & - & \\
\hline NGC 7187 & $(\mathrm{R}) \mathrm{SAB}(\mathrm{r}) 0^{+}$ & 13.57 & 41 & 26 & 134 & 33.8 & $\ldots$ & + & 2 \\
\hline NGC 7280 & $\mathrm{SAB}(\mathrm{r}) 0^{+}$ & 12.80 & 66 & 48 & 72 & 24.3 & 111 & $+?$ & \\
\hline NGC 7716 & SAB(r)b: & 12.63 & 64 & 34 & 35 & 34.1 & $\ldots$ & + & \\
\hline Mrk 573 & (R)SAB(rs) $0^{+}$: & 14.49 & 40 & 12 & $\ldots$ & 68.3 & 130 & - & \\
\hline UGC 524 & $\left(\mathrm{R}^{\prime}\right) \mathrm{SB}(\mathrm{s}) \mathrm{b}$ & 14.39 & 31 & 21 & 120 & 145.0 & $\ldots$ & + & \\
\hline ESO 215-G031 & $\left(\mathrm{R}_{1}^{\prime}\right) \mathrm{SB}(\mathrm{r}) \mathrm{b}$ & 12.18 & 70 & 42 & 130 & 33.6 & $\ldots$ & + & \\
\hline ESO 320-G030 & $\left(\mathrm{R}_{1}^{\prime}\right) \mathrm{SAB}(\mathrm{r}) \mathrm{a}$ & 13.16 & 67 & 54 & 121 & 40.1 & $\ldots$ & - & \\
\hline ESO 443-G039 & S0? & 13.43 & 40 & 58 & 14 & 39.1 & 132 & $\ldots$ & \\
\hline ESO 447-G030 & (R) $\mathrm{SAB}(\mathrm{rl}) 0^{+}$ & 12.98 & 43 & 50 & 35 & 37.6 & 163 & - & \\
\hline IRAS $03565+2139$ & SBa? & 14.91 & 17 & 20 & $\ldots$ & 101.0 & $\ldots$ & - & 3 \\
\hline
\end{tabular}

Column (1): galaxy name. Column (2): morphological type from NED (primarily from RC3). Column (3): total corrected $B$-band magnitude, from LEDA. Column (4): one-half of the $\mu_{B}=25$ diameter from RC3. Column (5): galaxy inclination. Column (6): position angle of galaxy major axis. Column (7): distance in Mpc. Column (8): central velocity dispersion. Column (9): sense of spiral arm rotation ("++" = clockwise, "“ -" = counter-clockwise). Column (10): notes for individual galaxies: $1=$ Triple-barred galaxy. $2=$ Also has an inner disk. $3=$ Morphological type is visual estimate (none listed in NED). Sources of data for individual galaxies are discussed in Sect. 5. 
Table 2. General data for barred galaxies with inner disks.

\begin{tabular}{|c|c|c|c|c|c|c|c|c|c|}
\hline Name & 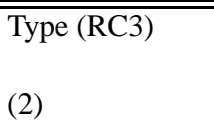 & 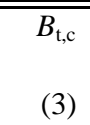 & $\begin{array}{r}R_{25} \\
\prime \\
(4)\end{array}$ & $\begin{array}{r}i \\
\circ \\
(5)\end{array}$ & $\begin{array}{r}\text { Disk PA } \\
\circ \\
(6)\end{array}$ & $\begin{array}{r}\text { Distance } \\
\mathrm{Mpc} \\
(7)\end{array}$ & $\begin{array}{r}\sigma_{0} \\
\mathrm{~km} \mathrm{~s}^{-1} \\
(8)\end{array}$ & Rot & $\begin{array}{r}\text { Notes } \\
\text { (10) }\end{array}$ \\
\hline NGC 151 & $\mathrm{SB}(\mathrm{r}) \mathrm{bc}$ & 11.65 & 111 & 65 & 75 & 48.9 & $\ldots$ & + & \\
\hline NGC 470 & $\mathrm{SA}(\mathrm{rs}) \mathrm{b}$ & 11.99 & 84 & 56 & 155 & 31.3 & 143 & + & \\
\hline NGC 1398 & $\left(\mathrm{R}_{1} \mathrm{R}_{2}^{\prime}\right) \mathrm{SB}(\mathrm{rs}) \mathrm{ab}$ & 10.29 & 212 & 45 & 96 & 16.1 & 205 & + & \\
\hline NGC 2787 & $\mathrm{SB}(\mathrm{r}) 0^{+}$ & 11.27 & 95 & 55 & 109 & 7.5 & 195 & + & \\
\hline NGC 2880 & $\mathrm{SB}^{-}$ & 12.32 & 62 & 52 & 144 & 21.9 & 142 & $-?$ & \\
\hline NGC 3266 & $\mathrm{SAB} 0^{0} ?$ & 13.58 & 46 & 35 & 85 & 25.1 & 116 & $\ldots$ & 1 \\
\hline NGC 3384 & $\mathrm{SB}(\mathrm{s}) 0^{0}:$ & 10.68 & 165 & 61 & 50 & 11.6 & 140 & $\ldots$ & \\
\hline NGC 3412 & $\mathrm{SB}(\mathrm{s}) 0^{0}$ & 11.29 & 109 & 52 & 153 & 11.3 & 108 & $\ldots$ & \\
\hline NGC 4143 & $\mathrm{SAB}(\mathrm{s}) 0^{0}$ & 11.70 & 69 & 59 & 144 & 15.9 & 270 & - & \\
\hline NGC 4262 & $\mathrm{SB}(\mathrm{s}) 0^{-} ?$ & 12.26 & 56 & 29 & 153 & 15.3 & 188 & $\ldots$ & \\
\hline NGC 4386 & $\mathrm{SAB} 0^{0}:$ & 12.47 & 74 & 48 & 140 & 27.0 & 197 & $\ldots$ & 1 \\
\hline NGC 4612 & $(\mathrm{R}) \mathrm{SAB} 0^{0}$ & 12.08 & 74 & 44 & 143 & 15.3 & 61 & $\ldots$ & \\
\hline NGC 4754 & $\mathrm{SB}(\mathrm{r}) 0^{-}:$ & 11.40 & 137 & 63 & 23 & 16.8 & 200 & $\ldots$ & 1 \\
\hline NGC 7007 & $\mathrm{SA}^{-}:$ & 12.90 & 57 & 55 & 2 & 37.6 & 145 & $\ldots$ & \\
\hline UGC 6062 & $\mathrm{SAB}(\mathrm{r}) 0^{0}$ & 13.29 & 36 & 55 & 25 & 35.7 & 178 & $\ldots$ & 1 \\
\hline ESO 378-G020 & $\mathrm{SB}(\mathrm{rs}) 0^{0} ?$ & 13.17 & 39 & 57 & 34 & 38.3 & 132 & $\ldots$ & 1 \\
\hline ESO 443-G017 & (R)SB(rl)0/a & 13.53 & 42 & 50 & 23 & 39.3 & $\ldots$ & - & \\
\hline
\end{tabular}

Columns are the same as for Table 1. As noted in Table 1, the double-barred galaxies NGC 3368, 3945, 4785, and 7187 also have inner disks; to save space, they are not repeated here. Notes for individual galaxies: 1 = unsharp masking suggests inner disk is probably an inner bar. Sources of data for individual galaxies are discussed in Sect. 5.

25th-magnitude values in de Vaucouleurs et al. (1991, hereafter RC3 $)^{2}$, and distances are based on the redshift listed in LEDA, corrected for Virgocentric motion (Paturel et al. 1997), and assuming a Hubble constant $H_{0}=75 \mathrm{~km} \mathrm{~s}^{-1} \mathrm{Mpc}^{-1}$. Similarly, central velocity dispersion $\sigma_{0}$ is from the compilation of McElroy (1995), unless otherwise noted.

NGC 151 (inner disk): Márquez et al. (1999), from which the bar measurements were taken. Inner-disk measurements are from a NICMOS3 F160W image, and agree well with the Márquez et al. values.

NGC 357: Mulchaey et al. (1997). Outer-bar measurements are from the publically available $K$-band image of Mulchaey et al.; inner-bar measurements are from a NICMOS2 F160W image. Outer disk inclination and PA are from ellipse fits to an unpublished, deep I-band image by Alfonso López Aguerri and Enrico Maria Corsini.

NGC 470 (inner disk): Wozniak et al. (1995) and Friedli et al. (1996). Bar and inner-disk measurements are based on the ellipse fits of Friedli et al. (1996); $\sigma_{0}$ is from Prugniel et al. (2001).

NGC 718: Erwin (2000); see Erwin \& Sparke (2003). Adopted inner-bar $L_{\mathrm{bar}}$ is that of the blue nuclear ring in Erwin \& Sparke (2003).

NGC 1068 (M 77): Schinnerer et al. (2000). Depending on how one counts, as many as five separate bars have been suggested for this galaxy. The bar universally agreed upon is that first found in the near-IR by Scoville et al. (1988), with $a \sim 17^{\prime \prime}$. Schinnerer et al. (2000) presented both imaging and kinematic evidence for a much larger, rounder bar outside;

\footnotetext{
${ }^{2}$ Inclination is derived from the axis ratio $b / a$, assuming an intrinsic disk thickness $c / a=0.2$.
}

this is the same as the "oval disk" discussed by Kormendy \& Norman (1979). This galaxy is thus similar to NGC 4736 (below): a fairly strong and large inner bar resides inside a very large, weak outer bar/oval disk. The measurements presented here are based on the images and ellipse fits of Alonso-Herrero et al. (1998) for the inner bar (with $L_{\mathrm{bar}}$ based on the size of the nuclear pseudo-ring surrounding it) and on the 2MASS $J$-band image for the outer bar. Distance, inclination, and outer-disk PA are from Schinnerer et al. and references therein.

Other studies have proposed additional, smaller bars for this galaxy, but the evidence for these is ambiguous or dubious. Rouan et al. (1998), using adaptive-optics, near-IR images and archival F547M WFPC2 images, suggested no fewer than three nested bars, all smaller than the well-known 17" bar (i.e., the inner bar in this catalog). At least some of these features are probably due to dust and scattered nuclear radiation, and possibly the radio jet as well (Weinberger et al. 1999; Bock et al. 2000). Finally, Laine et al. (2002) reported two bars: the wellknown IR bar, and a smaller bar with deprojected $a=1.7^{\prime \prime}$, possibly matching the middle of Rouan et al.'s three bars. But Schinnerer et al. found no evidence for a nuclear bar on those size scales, and argued instead for a warped molecular disk, which might explain some of the features seen by Rouan et al. and Laine et al.

NGC 1097: Shaw et al. (1993) and Buta \& Crocker (1993). Outer-bar measurements are from the 2MASS $H$-band image, except that $\epsilon_{\max }$ is from the $I$-band ellipse fits of Wozniak et al. (1995), which are higher resolution, and $L_{\mathrm{bar}}$ is from spiral arms trailing off the ends of the bar, as seen in the $7 \mu \mathrm{m}$ ISO image (Roussel et al. 2001, available via NED). Inner-bar measurements are based on those of Quillen et al. (1995) and the ellipse fits in Shaw et al. (1993) and Friedli et al. (1996). Outer-disk PA and inclination are from H I and emission-line 
kinematics (Ondrechen et al. 1989; Storchi-Bergman et al. 1996).

NGC 1241: Laine et al. (2002). Outer-bar measurements are from a NICMOS3 F160W image, with inner-bar measurements from a NICMOS2 F160W image; the inner-bar $a_{\epsilon}$ is based on an extremum in the ellipse-fit PA, and $L_{\mathrm{bar}}$ is from the size of the nuclear ring.

NGC 1291: de Vaucouleurs (1974); de Vaucouleurs (1975). Outer-bar measurements are from the DSS2 red image, except for bar PA from Buta (1995). Inner-bar measurements are from an $I$-band image (courtesy Oak-Kyung Park). Disk inclination is from H I kinematics (van Driel et al. 1988); the disk PA is undefined.

NGC 1317: Schweizer (1980). Outer-bar measurements are from the publically available $R$-band image of Marcum et al. (2001); inner-bar measurements are from the publically available $K$-band image of Mulchaey et al. (1997), except that $L_{\text {bar }}$ is from size of the inner $\mathrm{H} \alpha$ nuclear ring. The outer-disk inclination is from the axis ratio quoted in Buta \& Crocker (1993); the distance is a default value for the Fornax Cluster, from the HST Key Project study of Freedman et al. (2001).

NGC 1398 (inner disk): Erwin et al. (2004), based partly on Jungwiert et al. (1997). The inner "triaxial bulge" identified by Jungwiert et al. (see also Wozniak et al. 1995), with $a_{\epsilon} \approx 13^{\prime \prime}$, appears to be a large stellar nuclear ring; the inner disk listed here shows up in HST images. Bar measurements are from the ellipse fits of Wozniak et al. (1995) and Jungwiert et al. (1997); inner-disk measurements are from WFPC2 F606W and F814W images. Outer-disk inclination and PA are from HI kinematics (Moore \& Gottesman 1995).

NGC 1433: Sandage \& Brucato (1979) and Buta (1986). Outer-bar measurements are from the ellipse fits of Wozniak et al. (1995) and Buta et al. (2001), with $L_{\mathrm{bar}}$ from the size of the inner ring in Buta et al. Inner-bar measurements are from the ellipse-fits of Jungwiert et al. (1997), with $L_{\mathrm{bar}}$ from size of nuclear ring (Buta et al.). Outer-disk inclination and PA are from H I kinematics (Ryder et al. 1996).

NGC 1543: de Vaucouleurs (1975) and Sandage \& Brucato (1979). Outer-bar measurements are from a DSS image; innerbar measurements are from WFPC2 F814W images, with $L_{\text {bar }}$ from the size of the stellar nuclear ring. Outer disk inclination is from the axis ratio quoted in Buta \& Crocker (1993); distance is from Tonry et al. (2001, surface-brightness fluctuation). See Fig. 3.

NGC 1808: Kotilainen et al. (1996), Tacconi-Garman et al. (1996), and Jungwiert et al. (1997). Outer-bar measurements are from publically available $H$-band image of the OSU Bright Spiral Galaxy Survey (Eskridge et al. 2002); inner-bar measurements are from the ellipse fits of Jungwiert et al. (1997). Outer-disk inclination is based on the axis ratio of the outer spiral arms listed in Koribalski et al. (1993).

The outer bar in this galaxy is quite peculiar (similar to those of NGC 4725 and ESO 443-39), and its position angle is somewhat ill-defined.

NGC 2217: Jungwiert et al. (1997). Measurements for both bars are from the ellipse fits of Jungwiert et al., except that $L_{\mathrm{bar}}$ for outer bar is from Ohta et al. (1990), based on the decline of the bar-interbar intensity ratio ${ }^{3}$. Outer-disk inclination and PA are from stellar kinematics (Bettoni et al. 1990).

NGC 2642: Erwin et al. (2004). Bar measurements are from a NICMOS3 F160W image, with $L_{\text {bar }}$ for inner bar from size of the nuclear ring and $L_{\mathrm{bar}}$ for outer bar from size of the inner ring/spiral arms encircling the bar.

NGC 2646: Erwin et al. (2004). Bar measurements are from ellipse fits to an $R$-band image. Disk PA is unknown; RC3 gives nearly circular outer isophotes.

NGC 2681: Wozniak et al. (1995) and Erwin \& Sparke (1999). See Erwin \& Sparke (2003).

NGC 2787 (inner disk): Erwin (2000); see Erwin \& Sparke (2003). The inner disk is discussed in greater detail in Erwin et al. (2003).

NGC 2859: Kormendy $(1979,1982 a)$ and Wozniak et al. (1995). See Erwin \& Sparke (2003).

NGC 2880 (inner disk): Erwin (2000); see Erwin \& Sparke (2003).

NGC 2950: Kormendy $(1979,1982 a)$ and Wozniak et al. (1995). See Erwin \& Sparke (2003).

NGC 2962: Erwin (2000); see Erwin \& Sparke (2003).

NGC 3081: Buta (1990a). Outer-bar measurements are based on those of Friedli et al. (1996), Mulchaey et al. (1997), and Buta \& Purcell (1998); $L_{\mathrm{bar}}$ is from the size of the inner ring, measured in the publically available $K$-band image of Mulchaey et al. Measurements for inner bar are from a NICMOS2 F160W image; $L_{\mathrm{bar}}$ is from the size of the nuclear ring. Disk inclination and PA are from kinematic and photometric arguments in Buta \& Purcell.

NGC 3266 (inner disk): Erwin et al. (2004). Bar measurements are from WFPC2 F702W images (Rest et al. 2001); the outer disk orientation is from ellipse fits to the WFPC2 mosaic image, and $\sigma_{0}$ is from Wegner et al. (2003). Unsharp masking suggests that the inner disk is probably an inner bar.

NGC 3275: Mulchaey et al. (1997). Outer-bar measurements are from the publically available images of Mulchaey et al.; inner-bar measurements are from a NICMOS3 F160W image, with $L_{\mathrm{bar}}$ based on the nuclear ring size.

NGC 3358: Buta \& Crocker (1991, 1993). Bar measurements are from ellipse fits to $V$ - and $I$-band images (outer bar $a_{\epsilon}$ is from PA minimum), with outer disk position angle from ellipse fits to a $B$-band image, all kindly provided by Ron Buta. Outer disk inclination is from the axis ratio quoted in Buta \& Crocker (1993).

NGC 3368 (M 96; double bar + inner disk): Source: Jungwiert et al. (1997). The $H$-band image of Jungwiert et al. is not large enough to fully cover the outer bar; thus, the "double bar" they reported is actually the inner bar + an intermediate elliptical structure, identified here as an inner disk due to its low ellipticity and alignment with the outer disk. Outer-bar, innerdisk, and inner-bar measurements are from the publically available $K$-band image of Möllenhoff \& Heidt (2001), except that inner-bar $a_{\epsilon}$ is based on ellipse fits to a NICMOS2 F160W image and $L_{\mathrm{bar}}$ on the size of the nuclear ring. See Erwin (2004a)

\footnotetext{
3 Erwin \& Sparke (2003) found that the Ohta et al. measurements were a good match to their definition of $L_{\mathrm{bar}}$, and the ellipse fits for this galaxy do not provide useful constraints.
} 
for a discussion of the outer-disk orientation; distance is from Cepheid measurements (Freedman et al. 2001).

NGC 3384 (inner disk): Busarello et al. (1996). Outer-bar measurements are from the ellipse fits of Busarello et al.; $a_{\epsilon}$ in this case is actually the ellipticity minimum, which is also the extremum in the PA twist. Bar PA is from analysis of Busarello et al., which showed that the best ellipse-fit PA for the bar is $\sim 60^{\circ}$ away from the bar's true position angle. (The problems with the ellipse fits comes from the fact that this galaxy is highly inclined, and the bar is very close to the projected minor axis.) Inner disk measurements are from WFPC2 F814W and NICMOS2 F160W images. Distance is from Tonry et al. (2001, surface-brightness fluctuation), and $\sigma_{0}$ is the average of several recent measurements (Fisher 1997; Neistein et al. 1999; Gebhardt et al. 2003).

NGC 3393: Jungwiert et al. (1997), Alonso-Herrero et al. (1998), and Greusard et al. (2000). Bar measurements are based the publically available $K$-band image of Mulchaey et al. (1997), plus the near-IR ellipse fits of Jungwiert et al. and Greusard et al. Outer-disk inclination is from Kornreich et al. (1998); outer-disk PA is undefined due to the low inclination.

NGC 3412 (inner disk): Erwin (2000); see Erwin \& Sparke (2003).

NGC 3941: Erwin (2000); see Erwin \& Sparke (2003).

NGC 3945 (double bar + inner disk): Kormendy (1979, 1982a) and Wozniak et al. (1995). See Erwin \& Sparke (1999) and Erwin \& Sparke (2003); for a detailed study of the inner disk, see Erwin et al. (2003).

Wozniak et al. (1995) suggested this galaxy might be triply barred (it is listed as such by Moiseev 2001); however, Erwin \& Sparke (1999) used HST images to show that it was doublebarred, with a large inner disk dominating the region between the two bars. Similar bar-disk-bar systems are NGC 3368, NGC 4785, and NGC 7187.

NGC 4143 (inner disk): Erwin (2000); see Erwin \& Sparke (2003). Central velocity dispersion is from Di Nella et al. (1995).

NGC 4262 (inner disk): Shaw et al. (1995). See Erwin et al. (2004) for source of bar and disk measurements. The distance is a default value for the Virgo Cluster, from the HST Key Project study of Freedman et al. (2001).

NGC 4303 (M 61): Colina \& Wada (2000). Outer-bar measurements are from the publically available $K$-band image of Möllenhoff \& Heidt (2001), with $L_{\text {bar }}$ from a spiral arm crossing over the southern end of the bar; inner-bar measurements are from a NICMOS2 F160W image. Outer-disk inclination and PA are from the kinematic arguments of Schinnerer et al. (2001); the distance is the default Virgo Cluster distance (see note for NGC 4262).

The outer-bar $a_{\epsilon}$ is based on a minimum in the PA, since there is no clear ellipticity maximum within the bar; the ellipticity maximum at $a \approx 45^{\prime \prime}$ (e.g., Laine et al. 2002) is due to spiral arms outside the bar.

NGC 4314: Benedict et al. (1993). See Erwin \& Sparke (2003), and Erwin (2004a) for measurements of the outer bar; $\sigma_{0}$ is from Barth et al. (2002).

NGC 4321 (M 100): Pierce (1986), Shaw et al. (1995), Knapen et al. (1995a). Outer-bar measurements are from ellipse fits to the 2MASS $K$-band image, with $L_{\text {bar }}$ from spiral arms crossing the ends of the bar; inner-bar measurements are from the near-IR ellipse fits in Pérez-Ramírez et al. (2000). Outer-disk inclination and PA are from the Hi kinematics of Knapen et al. (1993); distance is from Cepheids (Freedman et al. 2001).

NGC 4340: Kormendy (1979) and Wozniak et al. (1995). Bar and outer-disk measurements are from an MDM $R$-band image obtained by Paul Schechter; inner-bar $L_{\mathrm{bar}}$ is from size of stellar nuclear ring; see Fig. 3 and Erwin et al. (2001). The distance is the default Virgo Cluster distance (see note for NGC 4262).

NGC 4386 (inner disk): Erwin (2000); see Erwin \& Sparke (2003). Unsharp masking suggests that the inner disk is probably an inner bar.

NGC 4503: Erwin et al. (2004). Bar measurements are from the $H$-band image of Jungwiert et al. (1997), courtesy Françoise Combes, for the outer bar and from a WFPC2 F702W image for the inner bar. The distance is the default for the Virgo Cluster (see note for NGC 4262).

NGC 4612 (inner disk): Erwin et al. (2004), based on Jungwiert et al. (1997). All measurements, including outerdisk orientation, are from an MDM $R$-band image, courtesy Paul Schechter; the outer-bar and outer-disk measurements agree well with measurements made using the $R$-band image of Frei et al. (1996), available via NED. Central velocity dispersion is from Wegner et al. (2003).

NGC 4725: Laine et al. (2002). Outer-bar measurements are from the $r$-band image of Frei et al. (1996) and a $K$-band image kindly provided by Johan Knapen, with $L_{\text {bar }}$ from the inner ring; the outer bar is rather peculiar (similar to those of NGC 1808 and ESO 443-39), and its position angle is not well defined. Inner-bar measurements are from a NICMOS2 F160W image. See Erwin (2004a) for a discussion of the outerdisk orientation; the distance is from Cepheid measurements (Freedman et al. 2001).

NGC 4736 (M 94): Shaw et al. (1993) and Möllenhoff et al. (1995). As with NGC 1068, the outer bar is rather weak, and has been termed an "oval disk" rather than a bar. However, there is ample kinematic evidence that it rotates and behaves dynamically like a bar (e.g., Möllenhoff et al. 1995; van Driel et al. 1996, and references therein). Outer disk PA is from the kinematics and modeling of Möllenhoff et al. and van Driel et al., with inclination being the average of photometric and kinematic inclinations from those studies and the H I observations of Mulder \& van Driel (1993). Distance is from Tonry et al. (2001, surface-brightness fluctuation).

NGC 4785 (double bar + inner disk): Márquez et al. (1999), Erwin et al. (2004). Bar and inner disk measurements are from a NICMOS2 F160W image and the ellipse fits of Márquez et al. (1999); outer-bar $L_{\mathrm{bar}}$ is from spiral arms/inner ring. I classify the "inner bar" of Márquez et al. as an inner disk; the inner bar listed here was noted by them as an "inner elongation" in their unsharp mask of the NICMOS image. The outer bar is somewhat weak and difficult to distinguish from the spiral arms outside it. The central velocity dispersion is from Oliva et al. (1999). 
NGC 4754 (inner disk): Kormendy (1979); Shaw et al. (1995). All measurements, including outer-disk orientation, are from WIYN $R$-band images (Erwin et al. 2004); distance is from Tonry et al. (2001, surface-brightness fluctuation). Unsharp masking suggests that the inner disk is probably an inner bar.

NGC 4984: Jungwiert et al. (1997). Bar measurements are from the ellipse fits and isophotes in Jungwiert et al., with $L_{\text {bar }}$ for the outer bar from the inner-ring size listed in Buta \& Crocker (1993). Because the galaxy has prominent inner and outer rings (the RC3 position angle is basically that of the inner ring/lens), the outer disk orientation is based on considerations of typical inner- and outer-ring orientations and axis ratios compared with those of the galaxy's rings, similar to the approach used in Erwin \& Sparke $(1999,2003)$ for other galaxies with strong outer rings.

NGC 5365: Mulchaey et al. (1997). Bar measurements are from ellipse fits to the publically available $K$-band image of Mulchaey et al., with $a_{\epsilon}$ for the inner bar from an extremum in the PA. Outer disk orientation is from LEDA.

NGC 5728: Shaw et al. (1993) and Buta \& Crocker (1993). Outer-bar measurements are from the ellipse fits of Wozniak et al. (1995) and Prada \& Gutiérrez (1999); inner-bar measurements are from the near-IR ellipse fits and isophotes of Shaw et al. (1993), except that $a_{\epsilon}$ and $\epsilon_{\max }$ are from the higherresolution I-band ellipse fits of Prada \& Gutiérrez. The outer disk orientation is based on discussion in Nagar \& Wilson (1999).

NGC 5850: Buta \& Crocker (1993). Bar measurements are from a Nordic Optical Telescope $I$-band image (from the BARS Project; Lourenso \& Beckman 2001) and the near-IR ellipse fits of Friedli et al. (1996). $L_{\mathrm{bar}}$ is from the inner ring (Prieto et al. 1997) for the outer bar and from the nuclear ring for the inner bar. Outer disk orientation is from the HI kinematic study of Higdon et al. (1998).

NGC 6654: Erwin (2000); see Erwin \& Sparke (2003).

NGC 6684: Phillips et al. (1996). Outer-bar measurements are from a WFPC2 F814W mosaic image, with $L_{\mathrm{bar}}$ from the size of the inner pseudo-ring; inner-bar measurements are from a NICMOS3 F160W image. Outer disk inclination and PA are from ellipse fits to an unpublished, deep $I$-band image by Alfonso López Aguerri and Enrico Maria Corsini; distance is from Tonry et al. (2001, surface-brightness fluctuation). See Fig. 3.

NGC 6782: Buta \& Crocker (1993) and Wozniak et al. (1995). Outer-bar measurements are based on the ellipse fits of Friedli et al. (1996), Jungwiert et al. (1997), and a WFPC2 F814W image; inner-bar measurements are from the F814W image (good agreement with ellipse-fit values from Friedli et al. and Jungwiert et al.). Outer-disk inclination and PA are from the isophotal analysis in Quillen \& Frogel (1997); $\sigma_{0}$ is from Idiart et al. (1996).

NGC 7007 (inner disk): Mulchaey et al. (1997). Bar and inner-disk measurements are from the publically available $K$ band image of Mulchaey et al.; $\sigma_{0}$ is from Wegner et al. (2003).

NGC 7098: Source Buta \& Crocker (1993) and Wozniak et al. (1995). Outer-bar measurements are from the ellipse fits and isophotes of Friedli et al. (1996); inner-bar measurements are from a WFPC2 F814W image (good agreement with ellipse-fit values from Friedli et al.). Outer-disk inclination is from the axis ratio quoted in Buta \& Crocker (1993).

NGC 7187 (double bar + inner disk): Buta (1990b) and Wozniak et al. (1995). Outer-disk inclination and PA are from Buta; bar measurements are based on those of Buta and Wozniak et al. (1995). Adopted outer-bar length is that of the inner ring surrounding it.

Wozniak et al. (1995) identified this a triple-barred galaxy; however, the middle "bar" is low enough in ellipticity and close enough in alignment to the outer disk to qualify as an inner disk, making this galaxy similar to NGC 3368, NGC 3945, and NGC 4785.

NGC 7280: Erwin (2000); see Erwin \& Sparke (2003).

NGC 7716: Laine et al. (2002). Outer-bar measurements are from the publically available $K$-band image of Mulchaey et al. (1997); inner-bar measurements are from a NICMOS2 F160W image.

Mrk 573: Capetti et al. (1996), Alonso-Herrero et al. (1998). Laine et al. (2002) suggested that this galaxy was triple-barred; however, the largest of their three bars, identified via ellipse fits, is actually an outer ring (e.g., Afanasiev et al. 1996). Outer-bar measurements are from the $J$-band ellipse fits of Alonso-Herrero et al. (1998), with inner-bar measurements from a NICMOS1 F160W image; $L_{\mathrm{bar}}$ for the inner bar is from the nuclear ring.

UGC 524: Pogge \& Martini (2002). Bar measurements are from a WFPC2 F814W image. Outer-disk inclination and PA are from LEDA.

UGC 6062 (inner disk): Rest et al. (2001). Based on ellipse fits to the WFPC2 F702W images; bar and inner-disk position angles are from inspection of the isophotes and unsharp masks. The outer disk inclination and PA are from LEDA. Unsharp masking suggests that the inner disk is probably an inner bar.

ESO 215-31: Greusard et al. (2000). Bar measurements are from the near-IR ellipse fits of Greusard et al.

ESO 320-30: Greusard et al. (2000). Bar measurements are from the near-IR ellipse fits of Greusard et al.

ESO 378-20 (inner disk): Rest et al. (2001). Bar and inner disk measurements are from WFPC2 F702W images, with outer disk orientation from ellipse fits to the WFPC2 mosaic image; $\sigma_{0}$ is from Wegner et al. (2003). Unsharp masking suggests that the inner disk is probably an inner bar.

ESO 443-17 (inner disk): Greusard et al. (2000). Bar measurements are from the near-IR ellipse and isophotes of Greusard et al.

ESO 443-39: Rest et al. (2001). Bar measurements are from WFPC2 F702W images; the outer bar is peculiar and difficult to measure accurately (similar to NGC 1808 and NGC 4725). Central velocity dispersion is from Wegner et al. (2003).

ESO 447-30: Rest et al. (2001). Bar measurements are from WFPC2 F702W images; $\sigma_{0}$ is from Wegner et al. (2003). The outer disk orientation is somewhat uncertain, since $D_{25}$ coincides with an outer ring; however, all three rings (nuclear, inner, and outer) share a common PA of $34-36^{\circ}$ and ellipticities of $0.32-0.37$. 
IRAS 03565+2139: Erwin et al. (2004). Based on $R$-band observations by Chris Conselice and Jay Gallagher with the $3.5 \mathrm{~m}$ WIYN Telescope. $L_{\text {bar }}$ values for the inner and outer bars are from the sizes of the nuclear and inner rings, respectively. The inclination is based on inverting the $H$-band Tully-Fisher relation (as given in Binney \& Merrifield 1998, p. 425), using the $H$-band magnitude and the H I width $W_{20}$ from van Driel et al. (1995). Note that van Driel et al. considered this to be a deviant from their T-F relation, but that conclusion was based on the assumption that the RC3 axis ratio was that of the outer disk; the $R$-band image shows that the RC3 axis ratio is actually that of the inner ring, which is most likely not intrinsically circular (e.g., Buta 1986, 1995).

\section{Unconfirmed or ambiguous inner bars and disks}

Here, I list galaxies for which the evidence for a double-bar or inner-disk system is ambiguous or uncertain. Better observations (typically, near-IR and/or higher resolution) are needed in order to make definitive judgments one way or the other. This also includes some galaxies which are probably double-barred, but for which useful measurements are lacking.

NGC 613: Suggested as double-barred by Jungwiert et al. (1997), using their $H$-band image. Inspection of WFPC2 images reveals a pronounced and very elliptical nuclear ring with strong star formation, having the same size, ellipticity, and orientation as the suggested inner bar. While unsharp masking of the $H$-band image (courtesy Françoise Combes) displays something like an inner bar feature, the bright ends of the "bar" are resolved into multiple star clusters in the HST images. This indicates that the feature is probably not an inner bar, but higherresolution near-IR (preferably $K$-band) images are needed to decide one way or the other.

NGC 1079: Listed by Moiseev (2001), on the basis of an ellipse-fit feature in Jungwiert et al. (1997). However, the ellipticity peak is very weak, and Jungwiert et al. preferred to attribute it to "bulge triaxiality".

NGC 1326: This is one of two double-barred galaxies originally discussed by de Vaucouleurs (1974); it has also been reported by Buta \& Crocker (1993) and Wozniak et al. (1995). Inspection of WFPC2 F814W images shows very strong dust lanes inside a dusty, star-forming nuclear ring, which combine to produce the illusion of an inner bar. Near-IR images are needed to determine if there might still be an inner bar inside the nuclear ring.

NGC 1353: Jungwiert et al. (1997) noted a possible inner bar, though they were doubtful given the galaxy's high $\operatorname{inclination}\left(i \approx 70^{\circ}\right)$. Based on its orientation, the ellipsefit peak noted by Jungwiert et al. is a candidate inner disk. Inspection of a WFPC2 F606W image shows a very dusty nuclear ring with $r \approx 5^{\prime \prime}$ and complex dust lanes inside; this raises the possibility that residual dust extinction could cause the ellipse peak seen in $H$. Higher resolution near-IR imaging is needed to determine what is responsible for the ellipticity peak.

NGC 1371: Listed by Moiseev (2001), with a reference to Wozniak et al. (1995); however, this galaxy is not in the latter study. Since the bar sizes listed by Moiseev match those reported by Wozniak et al. for NGC 1317 , this is probably an accidental transposition/duplication.

NGC 1415: García-Barreto \& Moreno (2000) reported an inner "stellar bar" in red continuum images of this barred Sa. Since the position angle they report $\left(\sim 150^{\circ}\right)$ is very close to the RC3 position angle $\left(148^{\circ}\right)$, this is a candidate inner disk. No archival images are available; 2MASS images also suggest an inner elliptical feature, but there are indications of isophote twists, so this might be a nuclear spiral instead. Higher-resolution near-IR images are needed.

NGC 2339: Suggested as double-barred by Laine et al. (2002). Inspection of WFPC2 F606W and NICMOS2 F110W and $\mathrm{F} 160 \mathrm{~W}$ images indicates that the nuclear region has both strong dust lanes and star formation; the "bar" detected by Laine et al. appears to be an IR-bright (star-forming?) ring, with at least two bright nuclei inside. Higher-resolution $K$-band images are probably needed to be certain there is no inner bar here.

NGC 2639: Suggested as double-barred by Márquez et al. (1999), though Laine et al. (2002) found no bars in their analysis of this galaxy. Inspection, ellipse fitting, and unsharp masking of the NICMOS2 and NICMOS3 F160W and WFPC2 F547M images shows some evidence for a weak bar with $a \sim 4-5^{\prime \prime}$ (note that this does not match either of Márquez et al.'s proposed bars), oriented almost parallel with the projected outer disk; its presence is primarily signaled by the appearance of two classic, curved leading-edge dust lanes and a weak feature in the $H$-band ellipse fits. These dust lanes curve together at $r \lesssim 1.5^{\prime \prime}$ and meet in a chaotic, dusty region. There is tenuous evidence for a very weak elliptical feature with $a \sim 1.0^{\prime \prime}$ in the $H$-band ellipse fits, but there is also some indication that the dust seen in the WFPC2 image is distorting the $H$-band isophotes.

NGC 2442: Suggested by Moiseev (2001), based on the suggestion of a triaxial bulge by Baumgart \& Peterson (1986); Jungwiert et al. (1997) list this in their "twisted isophotes" category. Inspection of their $H$-band isophotes suggests there may be a nuclear bar with $a \sim 2.5-3^{\prime \prime}$; higher-resolution observations are needed for confirmation and accurate measurements. The outer bar is peculiar and difficult to define; it is somewhat similar to those of NGC 1808 and NGC 4725.

NGC 2685: Erwin \& Sparke (2002, 2003) listed this among their inner-disk galaxies. While the inner disk identification is strong, identification of the "spindle" as a bar (rather than a highly inclined large-scale disk) is sufficiently uncertain that this should probably not be considered a barred galaxy (see the discussion in Erwin \& Sparke 2003).

NGC 2782: Suggested by Jogee et al. (1999). As they point out, the evidence for a nuclear bar is rather good, but the case for an outer bar (or outer oval, in this case) is uncertain.

NGC 2811: Suggested by Márquez et al. (1999). The nearIR ellipse fits of Jungwiert et al. (1997) indicate a possible inner ellipticity peak, though it is at the limits of their resolution. Given the alignment, it is a candidate inner disk, but without higher resolution images the detection remains dubious.

NGC 2935: Suggested by Jungwiert et al. (1997). Because Buta \& Crocker (1993) reported a nuclear ring in this galaxy, there is the possibility that the "inner bar" detection is due to, 
or affected by, the nuclear ring. This is a promising candidate, but no higher resolution images are available.

NGC 3504: Pérez-Ramírez et al. (2000) noted a possible inner bar in this SBb galaxy, but also an apparent double nucleus. This latter feature makes it difficult to be certain about the inner bar.

NGC 4253: Suggested as double-barred by Márquez et al. (1999), based on ellipse fits. Though these are at the probable limit of their resolution, they are plausible; however, the ellipse fits of Peletier et al. (1999) do not agree with this. Unfortunately, the NICMOS2 F160W image is marred by saturation and a very strong diffraction pattern from the Seyfert nucleus. The inner isophotes appear fairly round and aligned with the (outer?) bar. The diffraction spikes are less severe in the WFPC2 F606W image, where there is no obvious sign of an inner bar. Strong dust lanes visible in the optical image could be the cause of the ellipse fit variations seen by Márquez et al. (1999); they also prevent a clear determination using the optical image.

NGC 4274: Suggested as double-barred by Shaw et al. (1995). Their near-IR image strongly suggests an inner bar, almost perpendicular to the outer bar, but no measurements are available. Unfortunately, the available WFPC2 images (F555W) show that the central region is extremely dusty, so near-IR images are required for measurement of the inner bar.

NGC 4290: Suggested by Márquez et al. (1999), though they labeled it as "uncertain". Their near-IR ellipse fits show an ellipticity peak, but without a stationary position angle, so a nuclear spiral is a possiblity. Higher-resolution images are needed.

NGC 4594 (M 014): Emsellem \& Ferruit (2000) suggested that the Sombrero Galaxy might be a double-bar system, based on color maps and 2D kinematics. The extremely high inclination of this galaxy makes finding (and measuring) any bar difficult, and Emsellem \& Ferruit point to alternate explanations and emphasize the tentative nature of this identification. (Part of the evidence for the inner bar is an apparent straight dust lane, evoking the classic leading-edge dust lanes of large-scale bars; but Maciejewski et al. (2002) and Shlosman \& Heller (2002) argue, on the basis of hydrodynamical simulations of double bars, that straight dust lanes are probably not characteristic of inner bars.)

NGC 4750: Suggested as double-barred by Laine et al. (2002). Inspection of NICMOS2 images show a clear, strong nuclear bar; however, the presence of the outer bar is much less certain. Optical images (including WFPC2 F606W) are too dusty to be useful. 2MASS near-IR images show an elliptical region corresponding to the $a=14^{\prime \prime}$ outer bar of Laine et al.; however, unsharp masking shows that at least part of this structure is a tightly wrapped spiral/pseudo-ring. Higher-resolution and higher $\mathrm{S} / \mathrm{N}$ near-IR images are needed to determine if a bar is present in addition to the spirals, and to properly measure its size and orientation.

NGC 4941: Greusard et al. (2000) found a nuclear bar in their near-IR images of this SABab galaxy, but argued that there was no outer bar. On the other hand, Eskridge et al. (2002) classify the galaxy as SAB based on their low-resolution nearIR images, and Kormendy (1982b) includes it in his list of galaxies with "oval disks", so it may be similar to NGC 1068 and NGC 4736: a galaxy with a strong inner bar and a weak outer bar. For the time being, the evidence for an outer bar remains too tenuous to classify this as a double-barred galaxy.

NGC 5101: Suggested as a possible double bar by Jungwiert et al. (1997). However, as they pointed out, the ellipticity peak is very weak and close to their seeing limit. Unsharp masking of their $H$-band image (courtesy Françoise Combes) suggests a possible nuclear ring or spiral. No higher-resolution images are available.

NGC 5566: Suggested as double-barred by Jungwiert et al. (1997); the orientation of inner structure would make it an inner disk. However, unsharp masking of WFPC2 F606W images suggests that the inner elliptical feature is a pair of spiral arms. Due to the presence of numerous dust lanes, this is an uncertain classification; high-resolution near-IR images are needed to be certain one way or the other.

NGC 5905: Suggested as double-barred by Wozniak et al. (1995); the proposed inner bar is near the limits of their resolution, and could be due to the nuclear ring they also report. Higher-resolution images, preferably near-IR, are needed.

NGC 6155: Suggested as a possble double bar by Márquez et al. (1999). In this case, it is the outer bar whose existence is in doubt; Márquez et al. note that it could be spirals arms instead.

NGC 6860: Suggested as double-barred by Márquez et al. (1999), based on features in their near-IR ellipse fits and color maps. Because the ellipse-fit features $\left(a<2^{\prime \prime}\right)$ are only slightly larger than their seeing $\left(F W H M \approx 0.9^{\prime \prime}\right)$, this is unfortunately not a clear detection; higher-resolution images are clearly needed. (The available HST images are off-center or too low in signal to be useful.)

NGC 6907: Suggested as double-barred by Elmegreen et al. (1996). Examination of the publically available $B$-band and $H$-band images from the OSU Bright Spiral Galaxy Survey (Eskridge et al. 2002) shows a clear elliptical feature perfectly aligned with the outer disk, with apparently somewhat higher ellipticity (so this is not an inner disk). Unfortunately, available images do not have high enough resolution to rule out the possiblity of, e.g., a nuclear ring. The (outer?) bar is relatively short; ellipse-fit measurements are confused due to the strong spiral arms trailing off from the bar ends.

NGC 7742: Suggested as double-barred by Laine et al. (2002), though Wozniak et al. (1995) found no bars in their optical study. Inspection and unsharp masking of a NICMOS2 F160W image yields some evidence for a weak nuclear bar, matching the inner bar of Laine et al. Their outer bar, unfortunately, is difficult to confirm: it is either extraordinarily weak and round, or else an isophotal side effect of the bright, starforming ring and its associated dust lanes.

IC 454: Suggested as double-barred by Márquez et al. (1999), based on features in their near-IR ellipse fits. This is a promising candidate, but since the size of the apparent inner bar $\left(a=3^{\prime \prime}\right)$ is only a little larger than their resolution (seeing $\left.=1.2-1.3^{\prime \prime}\right)$, it should probably be confirmed with higher resolution observations.

IC 1816: Suggested as double-barred by Márquez et al. (1999), though they were uncertain about the inner bar's 
existence. Examination, ellipse fits, and unsharp masking of a WFPC2 F606W image indicates that the inner ellipticity peak is most likely due to a chaotic, possibly star-forming nuclear ring and associated dust lanes; high-resolution near-IR imaging needed to clearly determine if there is or is not an inner bar inside the nuclear ring.

IC 2510: Suggested as a possible double bar by Márquez et al. (1999); the inner bar detection was based on only one method, so they considered it "doubtful". The WFPC2 F606W image shows a chaotic morphology dominated by dust; higherresolution near-IR images are probably needed to determine if there is an inner bar in this galaxy.

Mrk 1066: Suggested as double-barred by Laine et al. (2002); also listed by Moiseev (2001), on the basis of optical images by Afanasiev et al. (1998). Inspection of NICMOS2 F160W and F205W images shows an elongated central region, matching the inner bar of Laine et al.; unfortunately, strong isophote twisting, dust lanes, and IR-bright spiral structure make it difficult to be certain this is a bar, rather than an inclined or distorted pair of spiral arms.

UGC 3223: Suggested as double-barred by Márquez et al. (1999), though they were uncertain about the inner bar's existence; their ellipse fits just barely suggest something might be there. Nothing barlike is apparent in a WFPC2 F606W image, though there are strong dust lanes. Higher-resolution infrared images are needed.

ESO 323-77: Greusard et al. (2000) listed this as a "bar + triaxial bulge" object; the orientation of the inner ellipticity peak suggests a possible inner disk $\left(\mathrm{PA}=156^{\circ}\right.$, versus $155^{\circ}$ for outer disk from RC3). Inspection of WFPC2 F606W images shows tightly wrapped spiral arms in the nuclear region, with some bright knots possibly indicating star formation. High-resolution near-IR imaging is needed to determine if the near-IR isophote twists seen by Greusard et al. are solely due to the spiral arms.

ESO 437-67: Suggested by Jungwiert et al. (1997). However, the proposed secondary bar is too small to be securely identified; higher resolution images needed, as Jungwiert et al. note.

ESO 508-78: Suggested by (Buta \& Crocker 1991, 1993). Inspection of images kindly provided by Ron Buta shows a possible elliptical feature in the galaxy center, but the resolution is not good enough to clearly determine whether it is an inner bar or not.

Milky Way: Alard (2001) recently presented evidence for an asymmetric planar structure in the central $2-3^{\circ}$ of the galactic center, seen in near-IR star counts from 2MASS after subtracting the contribution of the known, large-scale bar. The asymmetry suggests a different orientation than that of the large-scale bar, which might indicate a small bar seen at an an intermediate position angle. However, van Loon et al. (2003) argue, from an analysis of ISOGAL and DENIS data, that the inner $\sim 1 \mathrm{kpc}$ of the Galaxy is axisymmetric.

\section{Misclassified single-bar (or unbarred) galaxies}

The following list contains galaxies which have been suggested as being double-barred or inner-disk galaxies, but which a careful analysis (often using archival HST images) indicates are probably not. In many cases, there is a distinct feature inside a large-scale bar which masquerades as an inner bar, but it is a nuclear ring or nuclear spiral - or simply strong dust lanes - instead.

NGC 1300: Suggested as a possible double bar by Moiseev (2001), on the basis of optical plates in Baumgart \& Peterson (1986). Using ground-based and NICMOS near-IR images, Pérez-Ramírez et al. (2000) found a nuclear ring, but no evidence for an inner bar.

NGC 1365: Suggested as double-barred by Jungwiert et al. (1997) and Laine et al. (2002), using ground-based and NICMOS images, respectively. Inspection of the NICMOS images shows that the ellipticity peak is due to a nuclear ring, with no evidence for a bar inside; a similar conclusion was reached by Emsellem et al. (2001) based on stellar kinematics from long-slit spectroscopy.

NGC 1512: Listed by Moiseev (2001), on the basis of weak ellipse-fit features noted by Jungwiert et al. (1997) in their nearIR images (which they interpreted as evidence for a triaxial bulge). Examination of NICMOS2 archival images (F160W and $\mathrm{F} 187 \mathrm{~W}$ ) shows a narrow, star-forming nuclear ring, with no evidence for any bar inside. The slight PA and ellipticity variations seen by Jungwiert et al. are almost certainly due to the nuclear ring.

NGC 1530: Suggested as double-barred by Laine et al. (2002) on the basis of ellipse fits to NICMOS images. However, Pérez-Ramírez et al. (2000), using ground-based near-IR images, argued that a nuclear spiral is main inner feature. Inspection of the NICMOS2 F160W image and unsharp masks strongly suggests a nuclear spiral rather than a bar (see also Martini et al. 2003). There is some suggestion from both the F160W image and from the WFPC2 F606W images that the innermost spiral may be lopsided, with one arm brighter than the other.

NGC 1566: Listed by Moiseev (2001), based on the suggestion of a triaxial bulge by Baumgart \& Peterson (1986). Inspection of F606W and F814W WFPC2 images shows strong dust lanes leading to a nuclear spiral in the inner $r<3^{\prime \prime}$ region. The $K$-band image of Mulchaey et al. (1997) shows no inner ellipticity peak and only a slight twist in the position angle, most likely due to the nuclear spiral.

NGC 1667: Suggested as double-barred by Laine et al. (2002). Inspection of the $K$-band image of Mulchaey et al. (1997), the NICMOS2 F160W image, and the WFPC2 F606W image all indicate that "outer bar" is really a pair of spiral arms (as noted by Mulchaey et al.); see Fig. 6. In this case, it is the "inner" bar which is evidently the genuine (and only) bar in this galaxy; isophote twists interior to it are due to strong dust lanes.

NGC 1672: Listed by Moiseev (2001), based on the suggestion of a triaxial bulge by Baumgart \& Peterson (1986). Inspection of F814W WFPC2 and F160W NICMOS2 images show a broad, star-forming nuclear ring, with a nuclear spiral inside (see also Martini et al. 2003). Although the latter is dusty, the dust is confined to the eastern side; there is no sign of an inner bar. 
NGC 2273: Suggested as double-barred by Mulchaey et al. (1997). Optical and near-IR HST images show inner bar is nuclear ring with two-armed spiral inside (see Fig. 4 and Erwin \& Sparke 2003; Martini et al. 2003).

NGC 2712: Suggested as double-barred by Márquez et al. (1999), based on their near-IR images. In this case, inspection of unpublished, higher-resolution $J$ and $K$-band images from the William Herschel Telescope (Erwin et al., in prep.) shows that the inner elliptical feature identified by Márquez et al. is a somewhat irregular nuclear ring; there is no evidence for a bar inside this ring.

NGC 3359: Sempere (1999) and Rozas \& Sempere (2000) suggested that this galaxy might have two decoupled bars, based on attempts to match hydrodynamic simulations to $\mathrm{HI}$ and $\mathrm{H} \alpha$ morphology, and on evidence of isophote twisting inside the "main" bar in an $I$-band image. However, analysis of a deep $I$-band image from the BARS Project (Lourenso \& Beckman 2001) and of the $K$-band image of Möllenhoff \& Heidt (2001) shows evidence for only one (stellar) bar, with a radius of $\sim 15-20^{\prime \prime}$. The isophote twisting in the $I$ band is absent at $K$, and is almost certainly due to dust rather than to an inner bar. (This does not rule out multiple pattern speeds in the galaxy, however: the two pattern speeds reported by Rozas \& Sempere could be those of the bar and the outer spirals, respectively.)

NGC 3786: Listed in Moiseev (2001), on the basis of optical images in Afanasiev et al. (1998). However, Moiseev (2002) reported that the "outer bar" is really spiral arms, and there is no sign of any large-scale bar in the WFPC2 F606W image.

NGC 4371: Wozniak et al. (1995) suggested this galaxy might be triply barred (it is listed as such by Moiseev 2001), but pointed out that high inclination and alignment of inner two "bars" with outer disk could mean that they were projected axisymmetric structures; Kormendy (1979) noted a possible secondary bar in this galaxy, which corresponds to the middle of W95's three ellipticity peaks. Erwin \& Sparke (1999) showed that the middle ellipticity peak was due to a bright, stellar ring; the apparent inner peak was produced by the effects of superimposing a ring on a rounder bulge profile, accompanied by circularizing of the inner isophotes by seeing (see Fig. 5 and Erwin et al. 2001).

NGC 4593: Listed by Moiseev (2001), based on the suggestion of a triaxial bulge by Wozniak et al. (1995). The latter authors noted the presence of dust and a blue nuclear ring with $r \sim 2^{\prime \prime}$. Inspection of a NICMOS2 F160W confirms that the central region of this galaxy is dominated by spiral dust lanes surrounding a nuclear ring or pseudo-ring; there is no evidence for an inner bar.

NGC 4643: Listed as an "inner-disk" galaxy by Erwin \& Sparke (2003); higher-resolution $I$-band images (courtesy Johan Knapen) and unpublished $H$-band images from the William Herschel Telescope appear to confirm the alternate hypothesis of Erwin \& Sparke that this is a stellar nuclear ring instead of an inner disk.

NGC 5033: Suggested as a triple bar system by Laine et al. (2002). Martini et al. (2001) did not find any bars in their analysis of the same images, and Laurikainen et al. (2002) found no sign of a bar in their tangential-force analysis of this galaxy using 2MASS images. Inspection of HST NICMOS1 and NICMOS2 images (and a WFPC2 F606W image) shows extremely strong spiral dust lanes in a highly inclined galaxy, with small, luminous bulge in center (see also Martini et al. 2003). The $K$-band image of Peletier et al. (1999) does suggest that the outermost bar of Laine et al. might be real, but the inner ellipticity peaks are almost certainly due to dust.

NGC 5383: Suggested as a potential double bar by Regan $\&$ Elmegreen (1997) on basis of ground-based $K$-band images, though they noted possibility of "projection effects". Also reported as double-barred by Laine et al. (2002). However, Sheth et al. (2000), using NICMOS2 images, showed that the inner feature is a nuclear spiral.

NGC 6221: Suggested as double-barred by Greusard et al. (2000), based on ellipse fits to their ground-based near-IR images; the orientation is the same as the outer disk, so this would be an inner disk candidate. However, NICMOS2 F160W images indicate that a nuclear spiral is most likely the cause of the inner ellipticity peak.

NGC 6300: Both Mulchaey et al. (1997) and Laine et al. (2002) have suggested that this galaxy is double-barred (though with disagreements about the size of the inner bar). Inspection of the NIMCOS2 F160W image shows that the inner isophotes are indeed elongated and twisted; however, these are distortions caused by a strong dust lane (clearly visible in WFPC 2 images).

NGC 6951: Suggested as double-barred by Wozniak et al. (1995) and Mulchaey et al. (1997). However, Friedli et al. (1996) noted that it might be nuclear ring instead, and NICMOS2 F110W and F160W images confirm the latter interpretation (e.g., Pérez et al. 2000; Pérez-Ramírez et al. 2000; Martini et al. 2003).

NGC 7743: Regan \& Mulchaey (1999) suggested this as double-barred based on the appearance of straight dust lanes. However, Shlosman \& Heller (2002) and Maciejewski et al. (2002) have used hydrodynamical simulations to argue that inner bars are unlikely to have straight dust lanes, so identifications based solely on the shape of dust lanes are suspect. Erwin \& Sparke (2003) argued that the "straight" dust lanes were really part of a nuclear spiral.

Mrk 471: Suggested as double-barred by Martini \& Pogge (1999) and Martini et al. (2001), based on NICMOS1 F160W images. Inspection of both those images and the F110W images indicates that the isophote distortions (and the resulting ellipse fits) are due to strong, asymmetric dust extinction; there are no other indications of a barlike structure in the central regions.

IC 184: Suggested as double-barred by Márquez et al. (1999). Inspection of the WFPC2 F606W image shows the "inner bar" quite clearly as the galaxy's only bar; the "outer bar" is resolved into a series of bright spiral arms (thus, this galaxy is similar to NGC 1667, above). There is no sign of any bar inside the "inner" bar; there is an inner ellipticity peak in the WFPC2 ellipse fits, but this is apparently due to dust lanes (high-res near-IR imaging may be needed to clearly rule out a true inner bar).

UGC 1395: Suggested as double-barred by Márquez et al. (1999), on the basis of unsharp masking. There is no sign of any structure with the size and position angle they suggest in the F606W WFPC2 image, or in the NICMOS1 F160W images; 
strong dust lanes in the bar may be confusing things. Martini \& Pogge (1999) did not report finding a bar, and there is no evidence for a bar in published near-IR images of Peletier et al. (1999).

Acknowledgements. I would like to thank Marc Balcells, Linda Sparke, Andrew Cardwell, and John Beckman for interesting discussions and helpful comments on early drafts of the work. I am also grateful to several people who provided me with images of various candidate double-barred galaxies, including Ron Buta, Françoise Combes, Chris Conselice and Jay Gallagher, Johan Knapen, Alfonso López Aguerri and Enrico Maria Corsini, Oak-Kyoung Park, and Paul Schechter.

This work made use of data from the Ohio State University Bright Spiral Galaxy Survey, which was funded by grants AST-9217716 and AST-9617006 from the United States National Science Foundation, with additional support from Ohio State University. It also made use of images from the Barred and Ringed Spirals (BARS) database, for which time was awarded by the Comité Científico Internacional of the Canary Islands Observatories. Based on observations made with the NASA/ESA Hubble Space Telescope, obtained from the data archive at the Space Telescope Science Institute. STScI is operated by the Association of Universities for Research in Astronomy, Inc. under NASA contract NAS 5-26555.

Finally, this research made use of the Lyon-Meudon Extragalactic Database (LEDA; part of HyperLeda at http://leda.univlyon1.fr/), and the NASA/IPAC Extragalactic Database (NED), which is operated by the Jet Propulsion Laboratory, California Institute of Technology, under contract with the National Aeronautics and Space Administration.

\section{References}

Afanasiev, V. L., Burenkov, A. N., Shapovalova, A. I., \& Vlasyuk, V. V. 1996, in Barred Galaxies, ed. R. Buta, B. G. Elmegreen, \& D. A. Crocker (San Francisco: ASP), IAU Colloq., 157, 218

Afanasiev, V. L., Mikhailov, V. P., \& Shapovalova, A. I. 1998, Astron. Astrophys. Trans., 16, 257

Alard, C. 2001, A\&A, 379, L44

Alonso-Herrero, A., Simpson, C., Ward, M. J., \& Wilson, A. S. 1998, ApJ, 495, 196

Ann, H. B. 2001, AJ, 121, 2515

Athanassoula, E. 2003, MNRAS, 341, 1179

Athanassoula, E., \& Misiriotis, A. 2002, MNRAS, 330, 35

Barth, A. J., Ho, L. C., \& Sargent, W. L. W. 2002, AJ, 124, 2607

Baumgart, C. W., \& Peterson, C. J. 1986, PASP, 98, 56

Benedict, G. F., Higdon, J. L., Jeffreys, W. H., et al. 1993, AJ, 105, 1369

Bettoni, D., Fasano, G., \& Galletta, G. 1990, AJ, 99, 1789

Binney, J., \& Merrifield, M. 1998, Galactic Astronomy (Princeton: Princeton Univ. Press)

Bock, J. J., Neugebauer, G., Matthews, K., et al. 2000, AJ, 120, 2904

Busarello, G., Capaccioli, M., D’Onofrio, M., Longo, G., et al. 1996, A\&A, 314, 32

Buta, R. 1986, ApJS, 61, 631

Buta, R. 1995, ApJS, 96, 39

Buta, R. 1990a, ApJ, 351, 62

Buta, R. 1990b, ApJ, 354, 428

Buta, R. 1995, ApJS, 96, 39

Buta, R., \& Crocker, D. A. 1991, AJ, 102, 1715

Buta, R., \& Crocker, D. A. 1993, AJ, 105, 1344

Buta, R., \& Purcell, G. B. 1998, AJ, 115, 484

Buta, R., \& Block, D. L. 2001, ApJ, 550, 243
Buta, R., Ryder, S. D., Madsen, G. J., et al. 2001, AJ, 121, 225

Capetti, A., Axon, D. J., Macchetto, F., Sparkes, W. B., \& Boksenberg, A. 1996, ApJ, 469, 554

Carollo, C. M., Stiavelli, M., de Zeeuw, P. T., \& Mack, J. 1997, AJ, 114, 2366

Colina, L., \& Wada, K. 2000, ApJ, 529, 845

Combes, F. 1994, in Mass-Transfer Induced Activity in Galaxies, ed. I. Shlosman (Cambridge: Cambridge U. Press), 170

Davies, C. L., \& Hunter, J. H., Jr. 1997, ApJ, 484, 79

de Vaucouleurs, G. 1974, in The Formation and Dynamics of Galaxies, ed. J. R. Shakeshaft (Dordrecht: Reidel), Proc. IAU Symp., 58, 1

de Vaucouleurs, G. 1975, ApJS, 29, 193

de Vaucouleurs, G., de Vaucouleurs, A., Corwin, H. G., Buta, R. J., Paturel, G., \& Fouqué, P. 1991, Third Reference Catalogue of Bright Galaxies (New York: Springer-Verlag) (RC3)

Di Nella, H., Garcia, A. M., Garnier, R., \& Paturel, G. 1995, A\&AS, 113,151

Elmegreen, B. G., \& Elmegreen, D. M. 1985, ApJ, 288, 438

Elmegreen, D. M., Elmegreen, B. G., Chromey, F. R., Hasselbacher, D. A., \& Bissell, B. A. 1996, AJ, 111, 1880

El-Zant, A. A., \& Shlosman, I. 2003, ApJ, 595, L41

Emsellem, E., \& Ferruit, P. 2000, A\&A, 357, 111

Emsellem, E., Greusard, D., Combes, F., et al. 2001, A\&A, 368, 52

Erwin, P. 2000, Ph.D. Thesis, University of Wisconsin

Erwin, P. 2004a, MNRAS, submitted

Erwin, P. 2004b, in preparation (Paper II)

Erwin, P., \& Sparke, L. S. 1999, ApJ, 512, L37

Erwin, P., \& Sparke, L. S. 2002, AJ, 124, 65

Erwin, P., \& Sparke, L. S. 2003, ApJS, 146, 299

Erwin, P., Vega Beltrán, J. C., \& Beckman, J. E. 2001, in The central kpc of starbursts and AGN: the La Palma connection, ed. J. H. Knapen, J. E. Beckman, I. Shlosman, \& T. J. Mahoney, San Francisco, ASP Conf. Ser., 171

Erwin, P., Vega Beltrán, J. C., Graham, A. W., \& Beckman, J. E. 2003, ApJ, 597, 929

Erwin, P., et al. 2004, in preparation

Eskridge, P. B., Frogel, J. A., Pogge, R. W., et al. 2002, ApJS, 143, 73

Fisher, D. 1997, AJ, 113, 950

Freedman, W. L., Madore, B. F., Gibson, B. K., et al. 2001, ApJ, 553, 47

Frei, Z., Guhathakurta, P., Gunn, J. E., \& Tyson, J. A. 1996, AJ, 111, 174

Friedli, D., \& Martinet, L. 1993, A\&A, 277, 27

Friedli, D., Wozniak, H., Rieke, M., Martinet, L., \& Bratschi, P. 1996, A\&AS, 118, 461

Friedli, D. 1996, in Barred Galaxies, ed. R. Buta, B. G. Elmegreen, \& D. A. Crocker (San Francisco: ASP), IAU Colloq., 157, 378

García-Barreto, J. A., \& Moreno, E. 2000, ApJ, 529, 832

Gebhardt, K., Richston, D., Tremaine, S., et al. 2003, ApJ, 583, 92

Greusard, D., Friedli, D., Wozniak, H., Martinet, L., \& Martin, P. 2000, A\&AS, 145, 425

Higdon, J. L., Buta, R. J., \& Purcell, G. B. 1998, AJ, 115, 80

Idiart, T. P., de Freitas Pacheco, J. A., \& Costa, R. D. D. 1996, AJ, 112,2541

Jarvis, B. J., Dubath, P., Martinet, L., \& Bacon, R. 1988, A\&AS, 74, 513

Jogee, S., Kenney, J. D. P., \& Smith, B. J. 1999, ApJ, 526, 665

Jungwiert, B., Combes, F., \& Axon, D. J. 1997, A\&AS, 125, 479

Knapen, J. H., Cepa, J., Beckman, J. E., del Rio, M. S., \& Pedlar, A. 1993, ApJ, 416, 563

Knapen, J. H., Beckman, J. E., Shlosman, I., et al. 1995a, ApJ, 443, L73 
Knapen, J. H., Beckman, J. E., Heller, C. H., Shlosman, I., \& de Jong, R. S. 1995b, ApJ, 454, 623

Koribalski, B., Dahlem, M., Mebold, U., \& Brinks, E. 1993, A\&A, 268, 14

Kotilainen, J. K., Forbes, D. A., Moorwood, A. F. M., van der Werf, P. P., \& Ward, M. J. 1996, A\&A, 313, 771

Kormendy, J. 1979, ApJ, 227, 714

Kormendy, J. 1982a, ApJ, 257, 75

Kormendy, J. 1982b, in Morphology and Dynamics of Galaxies: Twelfth Advanced Course of the Swiss Society of Astronomy and Astrophysics, ed. L. Martinet, \& M. Mayor (Sauverny: Observatoire de Genève), 113

Kormendy, J., \& Norman, C. A. 1979, ApJ, 233, 539

Kornreich, D. A., Haynes, M. P., \& Lovelace, R. V. E. 1998, AJ, 116, 2154

Laine, S., Shlosman, I., Knapen, J. H., \& Peletier, R. F. 2002, ApJ, 567, 97

Laurikainen, E., Salo, H., \& Rautiainen, P. 2002, MNRAS, 331, 880

Loursenso, S., \& Beckman, J. E. 2001, Ap\&SS, 276, 1161

Lütticke, R., Dettmar, R.-J., \& Pohlen, M. 2000, A\&A, 362, 435

McElroy, D. B. 1995, ApJS, 100, 105

Maciejewski, W., \& Sparke, L. S. 1997, ApJ, 484, L117

Maciejewski, W., \& Sparke, L. S. 2000, MNRAS, 313, 745

Maciejewski, W., Teuben, P. J., Sparke, L. S., \& Stone, J. M. 2002, MNRAS, 329, 502

Marcum, P. M., O’Connell, R. W., Fanelli, M. N., et al. 2001, ApJS, 132,129

Márquez, I., Durret, F., Delgado, R. M., et al. 1999, A\&A, 140, 1

Martin, P. 1995, AJ, 109, 2428

Martini, P., \& Pogge, R. W. 1999, AJ, 118, 2646

Martini, P., Pogge, R. W., Ravindranath, S., \& An, J. H. 2001, ApJ, 562, 139

Martini, P., Regan, M. W., Mulchaey, J. S., \& Pogge, R. W. 2003, ApJS, 146, 353

Moiseev, A. V. 2001, Bull. Spec. Astrophys. Obs., 51, 140 [astro-ph/0111220]

Moiseev, A. V. 2002, AstL, 28, 755

Moiseev, A. V., Valdés, J. R., \& Chavushyan, V. H. 2002, in Galaxies: The Third Dimension, ed. M. Rosado, L. Binette, \& L. Arias (San Francisco: ASP), 161

Möllenhoff, C., \& Heidt, J. 2001, A\&A, 368, 16

Möllenhoff, C., Matthias, M., \& Gerhard, O. E. 1995, A\&A, 301, 359

Moore, E. M., \& Gottesman, S. T. 1995, ApJ, 447, 159

Mulchaey, J. S., Regan, M. W., \& Kundu, A. 1997, ApJS, 110, 299

Mulder, P. S., \& van Driel, W. 1993, A\&A, 272, 63

Nagar, N. M., \& Wilson, A. S. 1999, ApJ, 516, 97

Neistein, E., Maoz, D., Rix, H.-W., \& Tonry, J. L. 1999, AJ, 117, 2666

Nieto, J.-L., Bender, R., Poulain, P., \& Surma, P. 1992, A\&A, 257, 97

Ohta, K., Hamabe, M., \& Wakamatsu K.-I. 1990, ApJ, 357, 71

Oliva, E., Origlia, L., Maiolino, R., \& Moorwood, A. F. M. 1999, A\&A, 350, 9

Ondrechen, M. P., van der Hulst, J. M., \& Hummel, E. 1989, ApJ, 342, 390

Prada, F., \& Gutiérrez, C. M. 1999, ApJ, 517, 123

Paturel, G., Andernach, H., Bottinelli, L., et al. 1997, A\&AS, 124, 109

Peletier, R. F., Knapen, J. H., Shlosman, I., et al. 1999, ApJS, 125, 363

Pérez, E., Márquez, I., Marrero, I., et al. 2000, A\&A, 353, 893

Pérez-Ramírez, D., Knapen, J. H., Peletier, R. F., et al. 2000, MNRAS, 317,234

Petitpas, G. R., \& Wilson, C. D. 2002, ApJ, 575, 814

Petitpas, G. R., \& Wilson, C. D. 2003, ApJ, 587, 649

Pfenniger, D., \& Norman, C. 1990, ApJ, 363, 391
Phillips, A. C., Illingworth, G. D., MacKenty, J. W., \& Franx, M. 1996, AJ, 111, 1566

Pierce, M. J. 1986, AJ, 92, 285

Pogge, R. W., \& Martini, P. 2002, ApJ, 569, 624

Prieto, M., Gottesman, S. T., Aguerri, A. L., \& Varela, A.-M. 1997, AJ, 114, 1413

Prugniel, Ph., Maubon, G., \& Simien, F. 2001, A\&A, 366, 68

Quillen, A. C., Frogel, J. A., Kuchinski, L. E., \& Terndrup, D. M. 1995, AJ, 110, 156

Quillen, A. C., \& Frogel, J. 1997, ApJ, 487, 603

Rautiainen, P., \& Salo, H. 1999, A\&A, 348, 737

Rautiainen, P., Salo, H., \& Laurikainen, E. 2002, MNRAS, 337, 1233

Regan, M. W., \& Elmegreen, D. M. 1997, AJ, 114, 965

Regan, M. W., \& Mulchaey, J. S. 1999, AJ, 117, 2676

Rest, A., van den Bosch, F. C., Jaffe, W., Tran, et al. 2001, AJ, 121, 2431

Rouan, D., Rigaut, F., Alloin, et al. 1998, A\&A, 339, 687

Roussel, H., Vigroux, L., Bosma, A., et al. 2001, A\&A, 369, 473

Rozas, M., \& Sempere, M. J. 2000, in Cosmic Evolution and Galaxy Formation: Structure, Interactions, and Feedback, The 3rd Guillermo Haro Astrophysics Conference, ed. J. Franco, L. Terlevich, O. López-Cruz, \& I. Aretxaga (San Francisco: ASP), 117

Ryder, S. D., Buta, R. J., Toledo, H., Shukla, H., et al. 1996, ApJ, 460, 665

Sandage, A., \& Brucato, R. 1979, AJ, 84, 472

Schinnerer, E., Eckart, A., Tacconi, L. J., Genzel, R., \& Downes, D. 2000, ApJ, 533, 850

Schinnerer, E., Maciejewski, W., Scoville, N., \& Moustakas, L. A. 2001, ApJ, 575, 826

Scoville, N. Z., Matthews, K., Carico, D. P., \& Sanders, D. B. 1988, ApJ, 327, L61

Schweizer, F. 1980, ApJ, 237, 303

Sempere, M. 1999, Ap\&SS, 269, 665

Shaw, M., Combes, F., Axon, D. J., \& Wright, G. S. 1993, A\&A, 273, 31

Shaw, M., Axon, D., Probst, R., \& Gatley, I. 1995, MNRAS, 274, 369

Sheth, K., Regan, M. W., Vogel, S. N., \& Teuben, P. J. 2000, ApJ, 532, 221

Shlosman, I., Frank, J., \& Begelman, M. C. 1989, Nature, 338, 45

Shlosman, I., \& Heller, C. H. 2002, ApJ, 565, 921

Storchi-Bergman, T., Wilson, A. S., \& Baldwin, J. A. 1996, ApJ, 460, 252

Tacconi-Garman, L. E., Sternberg, A., \& Eckart, A. 1996, AJ, 112, 918

Tonry, J. L., Dressler, A., Blakeslee, J. P., Ajhar, et al. 2001, ApJ, 546, 681

van den Bosch, F., \& Emsellem, E. 1998, MNRAS, 298, 267

van Driel, W., Rots, A. H., \& van Woerden, H. 1988, A\&A, 204, 39

van Driel, W., van den Broek, A. C., \& Baan, W. A. 1995, ApJ, 444, 80

van Driel, W., Mulder, P., \& Combes, F. 1996, in IAU Colloquium 157: Barred Galaxies, ed. R. Buta, B. G. Elmegreen, \& D. A. Crocker (San Francisco: ASP), 253

van Loon, J. Th., et al. 2003, MNRAS, 338, 857

Wegner, G., Bernardi, M., Wilmer, C. N. A., et al. 2003, AJ, in press [astro-ph/0308357]

Weinberger, A. J., Neugebauer, G., \& Matthews, K. 1999, AJ, 117, 2748

Wozniak, H., \& Pierce, M. J. 1991, A\&AS, 88, 325

Wozniak, H., Friedli, D., Martinet, L., Martin, P., \& Bratschi, P. 1995, A\&A, 111, 115 (W95) 


\section{Online Material}


Table 3. Measured values for bars and inner disks.

\begin{tabular}{|c|c|c|c|c|c|c|c|c|}
\hline Name & $\begin{array}{r}\text { Outer Disk PA } \\
\circ\end{array}$ & $\begin{array}{r}\text { Bar/Disk PA } \\
\text { 。 }\end{array}$ & $\begin{array}{r}a_{\epsilon} \\
\prime \prime\end{array}$ & $\begin{array}{c}a_{\min } \\
\prime \prime\end{array}$ & $\begin{array}{r}a_{10} \\
\prime \prime\end{array}$ & $\begin{array}{r}L_{\mathrm{bar}} \\
\prime \prime\end{array}$ & $\overline{\bar{\epsilon}}$ & NR? \\
\hline (1) & (2) & (3) & $(4)$ & $(5)$ & (6) & $(7)$ & (8) & (9) \\
\hline \multicolumn{9}{|c|}{ Double-Barred Galaxies } \\
\hline \multirow[t]{2}{*}{ NGC 357} & 20 & 120 & 21 & 28 & 27 & 27 & 0.44 & \\
\hline & & 45 & 3.1 & 5.7 & 4.4 & 4.4 & 0.16 & \\
\hline \multirow[t]{2}{*}{ NGC 718} & 5 & 152 & 20 & 33 & 30 & 30 & 0.23 & $\mathrm{Y}$ \\
\hline & & 15 & 1.6 & 4.0 & 3.6 & 3.3 & 0.19 & \\
\hline \multirow[t]{2}{*}{ NGC 1068} & 98 & 12 & 54 & 75 & 89 & 75 & 0.24 & $\mathrm{Y}$ \\
\hline & & 47 & 15 & 27 & $\cdots$ & 17 & 0.45 & \\
\hline \multirow[t]{2}{*}{ NGC 1097} & 134 & 147 & 88 & 120 & $\ldots$ & 107 & 0.67 & $\mathrm{Y}$ \\
\hline & & 30 & 7.5 & 8.1 & 7.7 & 7.7 & 0.46 & \\
\hline \multirow[t]{2}{*}{ NGC 1241} & 145 & 110 & 18 & 24 & 23 & 18 & 0.60 & $\mathrm{Y}$ \\
\hline & & 0 & 1.5 & $\ldots$ & 2.1 & 1.8 & 0.31 & \\
\hline \multirow[t]{2}{*}{ NGC 1291} & $\ldots$ & 171 & 89 & 130 & 140 & 130 & 0.39 & \\
\hline & & 15 & 18 & 26 & 24 & 24 & 0.24 & \\
\hline \multirow[t]{2}{*}{ NGC 1317} & 78 & 150 & 41 & 59 & 58 & 58 & 0.27 & $\mathrm{Y}$ \\
\hline & & 56 & 6.3 & 11 & 9.7 & 6.4 & 0.44 & \\
\hline \multirow[t]{2}{*}{ NGC 1433} & 21 & 97 & 74 & 110 & 150 & 90 & 0.70 & $\mathrm{Y}$ \\
\hline & & 32 & 6.2 & 13 & 12 & 12 & 0.38 & \\
\hline \multirow[t]{2}{*}{ NGC 1543} & $\ldots$ & 92 & 63 & 95 & $\ldots$ & 95 & 0.49 & $\mathrm{Y}$ \\
\hline & & 26 & 7.9 & 12 & 12 & 11 & 0.29 & \\
\hline \multirow[t]{2}{*}{ NGC 1808} & 133 & 144 & 80 & 114 & $\ldots$ & 114 & 0.66 & Y \\
\hline & & 158 & 3.3 & 5.9 & 4.8 & 4.8 & 0.53 & \\
\hline \multirow[t]{2}{*}{ NGC 2217} & 5 & 136 & 37 & $\ldots$ & $\ldots$ & 50 & 0.47 & \\
\hline & & 112 & 7.8 & 11 & 14 & 11 & 0.19 & \\
\hline \multirow[t]{2}{*}{ NGC 2642} & 140 & 115 & 25 & $\ldots$ & 29 & 26 & 0.64 & $\mathrm{Y}$ \\
\hline & & 145 & 1.5 & 2.7 & 2.7 & 2.0 & 0.30 & \\
\hline \multirow[t]{2}{*}{ NGC 2646} & $\ldots$ & 82 & 16 & 21 & 21 & 21 & 0.48 & \\
\hline & & 8 & 2.2 & 4.6 & 3.5 & 3.5 & 0.25 & \\
\hline \multirow[t]{3}{*}{ NGC 2681} & 140 & 30 & 50 & 75 & 60 & 60 & 0.23 & Y \\
\hline & & 73 & 18 & 23 & 19 & 19 & 0.33 & \\
\hline & & 20 & 1.7 & 3.9 & 3.3 & 3.3 & 0.26 & \\
\hline \multirow[t]{2}{*}{ NGC 2859} & 90 & 162 & 34 & 52 & 43 & 43 & 0.40 & Y \\
\hline & & 62 & 4.1 & 11 & 6.2 & 6.2 & 0.31 & \\
\hline \multirow[t]{2}{*}{ NGC 2950} & 120 & 162 & 24 & 41 & 31 & 31 & 0.43 & Y \\
\hline & & 85 & 3.2 & 6.3 & 3.9 & 3.9 & 0.33 & \\
\hline \multirow[t]{2}{*}{ NGC 2962} & 10 & 168 & 29 & 43 & $\ldots$ & 43 & 0.30 & \\
\hline & & 93 & 3.5 & $\ldots$ & 4.2 & 4.2 & 0.03 & \\
\hline
\end{tabular}

Observed position angles, sizes, and ellipticities for bars and inner disks of double-barred and inner-disk galaxies. For each galaxy, the first line contains values for the outer bar and the second line contains values for the inner bar (of double-barred galaxies) or inner disk. Column (1): galaxy name. Column (2): position angle of the outer disk, from Tables 1 or 2. Column (3): bar/disk position angle. Column (4): bar/disk semimajor axis of maximum isophotal ellipticity $a_{\epsilon}$ (lower limit on bar size). Column (5): semi-major axis of minimum ellipticity $a_{\min }$ outside $a_{\epsilon}$. Column (6): semi-major axis where $\mid \mathrm{PA}-$ bar PA $\mid>10^{\circ}$ outside $a_{\epsilon}$. Column (7): $L_{\text {bar }}$ (upper limit on bar size - usually the minimum of $a_{\text {min }}$ and $a_{10}$; see Sect. 5 for sources of alternate values). Column (8): maximum isophotal ellipticity $(\epsilon=1-b / a)$ of the bar or inner disk. Column (9): nuclear ring present? Note that NGC 3368, 3945, 4785, and 7187 have both inner bars and inner disks, and are thus included in each section. 
Peter Erwin: Double-barred galaxies. I., Online Material p 3

Table 3. continued.

\begin{tabular}{|c|c|c|c|c|c|c|c|c|}
\hline Name & $\begin{array}{c}\text { Outer Disk PA } \\
\circ\end{array}$ & $\begin{array}{r}\text { Bar/Disk PA } \\
\text { 。 }\end{array}$ & $\begin{array}{l}a_{\epsilon} \\
\prime \prime\end{array}$ & $\begin{array}{c}a_{\min } \\
\prime \prime\end{array}$ & $\begin{array}{r}a_{10} \\
\prime \prime\end{array}$ & $\begin{array}{r}L_{\mathrm{bar}} \\
\prime \prime\end{array}$ & 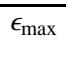 & $\overline{\mathrm{NR} ?}$ \\
\hline (1) & (2) & (3) & (4) & (5) & (6) & (7) & (8) & (9) \\
\hline \multirow[t]{2}{*}{ NGC 3081} & 97 & 69 & 33 & 41 & $\ldots$ & 35 & 0.65 & $\bar{Y}$ \\
\hline & & 122 & 5.7 & 8.6 & 7.1 & 5.8 & 0.50 & \\
\hline \multirow{2}{*}{ NGC 3275} & 122 & 120 & 28 & 36 & 48 & 36 & 0.58 & Y \\
\hline & & 172 & 2.1 & 3.5 & 2.7 & 2.6 & 0.31 & \\
\hline \multirow[t]{2}{*}{ NGC 3358} & 125 & 98 & 16 & 21 & 24 & 21 & 0.42 & \\
\hline & & 136 & 4.9 & 8.3 & 11 & 8.3 & 0.32 & \\
\hline \multirow[t]{2}{*}{ NGC 3368} & 172 & 115 & 61 & 80 & 75 & 75 & 0.40 & $\mathrm{Y}$ \\
\hline & & 129 & 3.4 & 6.8 & 5.9 & 5.0 & 0.35 & \\
\hline \multirow[t]{2}{*}{ NGC 3393} & $\ldots$ & 157 & 13 & 16 & 23 & 16 & 0.44 & \\
\hline & & 146 & 1.9 & 3.5 & 3.1 & 3.1 & 0.20 & \\
\hline \multirow[t]{2}{*}{ NGC 3941} & 10 & 166 & 21 & 36 & 32 & 32 & 0.47 & \\
\hline & & 85 & 3.2 & 4.7 & 4.4 & 4.4 & 0.21 & \\
\hline \multirow[t]{2}{*}{ NGC 3945} & 158 & 72 & 32 & 41 & 39 & 39 & 0.29 & Y \\
\hline & & 90 & 2.6 & 3.0 & 3.0 & 3.0 & 0.11 & \\
\hline \multirow[t]{2}{*}{ NGC 4303} & 138 & 179 & 29 & 50 & 48 & 34 & 0.50 & $\mathrm{Y}$ \\
\hline & & 40 & 1.8 & 2.8 & 2.5 & 2.5 & 0.29 & \\
\hline \multirow[t]{2}{*}{ NGC 4314} & 65 & 146 & 67 & 90 & 111 & 80 & 0.64 & $\mathrm{Y}$ \\
\hline & & 136 & 4.5 & 5.8 & 5.6 & 5.6 & 0.23 & \\
\hline \multirow[t]{2}{*}{ NGC 4321} & 153 & 107 & 55 & 61 & 80 & 58 & 0.54 & $\mathrm{Y}$ \\
\hline & & 110 & 8.2 & 15 & 10 & 10 & 0.62 & \\
\hline \multirow[t]{2}{*}{ NGC 4340} & 95 & 31 & 39 & 51 & 48 & 48 & 0.39 & $\mathrm{Y}$ \\
\hline & & 25 & 3.4 & 5.1 & 4.7 & 4.5 & 0.11 & \\
\hline \multirow[t]{2}{*}{ NGC 4503} & 12 & 6 & 23 & 27 & $\ldots$ & 27 & 0.48 & \\
\hline & & 45 & 2.9 & 4.8 & 6.0 & 4.8 & 0.25 & \\
\hline \multirow{2}{*}{ NGC 4725} & 40 & 50 & 118 & 130 & 170 & 125 & 0.67 & \\
\hline & & 141 & 5.6 & 7.3 & 6.8 & 6.8 & 0.20 & \\
\hline \multirow[t]{2}{*}{ NGC 4736} & 113 & 90 & 125 & 170 & $\ldots$ & 170 & 0.23 & Y \\
\hline & & 25 & 11 & 21 & 20 & 20 & 0.22 & \\
\hline \multirow[t]{2}{*}{ NGC 4785} & 81 & 66 & 11 & 13 & 16 & 12 & 0.57 & $\mathrm{Y}$ \\
\hline & & 127 & 0.9 & 1.1 & 1.2 & 1.1 & 0.21 & \\
\hline \multirow[t]{2}{*}{ NGC 4984} & 15 & 95 & 30 & $\ldots$ & 38 & 32 & 0.30 & Y \\
\hline & & 64 & 4.0 & 5.0 & 4.9 & 4.9 & 0.23 & \\
\hline \multirow[t]{2}{*}{ NGC 5365} & 4 & 108 & 25 & 33 & 31 & 31 & 0.25 & $\mathrm{Y}$ \\
\hline & & 35 & 3.7 & 16 & 5.3 & 5.3 & 0.28 & \\
\hline \multirow[t]{2}{*}{ NGC 5728} & 2 & 33 & 56 & 71 & $\ldots$ & 71 & 0.71 & $\mathrm{Y}$ \\
\hline & & 86 & 1.8 & 4.2 & 3.7 & 3.7 & 0.49 & \\
\hline \multirow[t]{2}{*}{ NGC 5850} & 163 & 116 & 63 & 84 & $\ldots$ & 75 & 0.68 & $\mathrm{Y}$ \\
\hline & & 50 & 5.9 & 9.2 & 7.6 & 7.1 & 0.30 & \\
\hline \multirow[t]{2}{*}{ NGC 6654} & 0 & 17 & 26 & 47 & 38 & 38 & 0.51 & \\
\hline & & 135 & 2.7 & 4.4 & 4.2 & 4.2 & 0.15 & \\
\hline \multirow[t]{2}{*}{ NGC 6684} & 36 & 150 & 26 & 33 & 29 & 28 & 0.25 & \\
\hline & & 68 & 2.9 & 6.2 & 4.1 & 4.1 & 0.35 & \\
\hline \multirow[t]{2}{*}{ NGC 6782} & 45 & 178 & 25 & 30 & 33 & 30 & 0.54 & $\mathrm{Y}$ \\
\hline & & 147 & 3.4 & 4.5 & 4.3 & 3.8 & 0.47 & \\
\hline
\end{tabular}


Peter Erwin: Double-barred galaxies. I., Online Material p 4

Table 3. continued.

\begin{tabular}{|c|c|c|c|c|c|c|c|c|}
\hline Name & $\begin{array}{r}\text { Outer Disk PA } \\
\circ\end{array}$ & $\begin{array}{r}\text { Bar/Disk PA } \\
\text { 。 }\end{array}$ & $\begin{array}{l}a_{\epsilon} \\
\prime \prime\end{array}$ & $\begin{array}{l}a_{\min } \\
\prime \prime\end{array}$ & $\begin{array}{r}a_{10} \\
\prime \prime\end{array}$ & $\begin{array}{r}L_{\mathrm{bar}} \\
\prime \prime\end{array}$ & $\overline{\epsilon_{\max }}$ & $\overline{\mathrm{NR} ?}$ \\
\hline (1) & (2) & (3) & (4) & (5) & (6) & (7) & (8) & (9) \\
\hline \multirow[t]{2}{*}{ NGC 7098} & 75 & 46 & 38 & 53 & 56 & 53 & 0.57 & \\
\hline & & 71 & 9.1 & 12 & 18 & 12 & 0.32 & \\
\hline \multirow[t]{2}{*}{ NGC 7187} & 134 & 66 & 19 & 28 & 22 & 19 & 0.37 & $\mathrm{Y}$ \\
\hline & & 54 & 2.5 & 3.8 & 3.5 & 3.5 & 0.19 & \\
\hline \multirow[t]{2}{*}{ NGC 7280} & 72 & 55 & 11 & 29 & 27 & 27 & 0.40 & \\
\hline & & 115 & 1.2 & 2.2 & 1.6 & 1.6 & 0.30 & \\
\hline \multirow[t]{2}{*}{ NGC 7716} & 35 & 25 & 22 & 28 & 25 & 25 & 0.35 & \\
\hline & & 57 & 3.1 & 5.4 & 5.6 & 5.4 & 0.38 & \\
\hline \multirow{2}{*}{ Mrk 573} & $\ldots$ & 0 & 9.0 & 12 & $\ldots$ & 12 & 0.33 & $\mathrm{Y}$ \\
\hline & & 83 & 1.2 & 3.1 & $\ldots$ & 2.1 & 0.31 & \\
\hline \multirow[t]{2}{*}{ UGC 524} & 120 & 143 & 8.5 & 11 & 12 & 11 & 0.54 & $\mathrm{Y}$ \\
\hline & & 167 & 1.0 & 1.1 & 1.3 & 1.1 & 0.43 & \\
\hline \multirow[t]{2}{*}{ ESO 215-G031 } & 130 & 147 & 38 & $\ldots$ & $\ldots$ & 47 & 0.63 & $\mathrm{Y}$ \\
\hline & & 153 & 5.3 & 9.5 & 6.2 & 6.2 & 0.48 & \\
\hline \multirow[t]{2}{*}{ ESO 320-G030 } & 121 & 142 & 23 & $\ldots$ & 41 & 37 & 0.64 & \\
\hline & & 107 & 3.9 & 5.2 & 4.5 & 4.5 & 0.32 & \\
\hline \multirow[t]{2}{*}{ ESO 443-G039 } & 14 & 27 & 11 & 19 & 25 & 19 & 0.51 & \\
\hline & & 44 & 1.8 & 2.4 & 2.7 & 2.4 & 0.24 & \\
\hline \multirow[t]{2}{*}{ ESO 447-G030 } & 35 & 133 & 13 & 15 & 14 & 14 & 0.17 & $\mathrm{Y}$ \\
\hline & & 177 & 2.8 & 9.7 & 3.6 & 3.6 & 0.36 & \\
\hline \multirow[t]{2}{*}{ IRAS $03565+2139$} & $\ldots$ & 4 & 13 & 18 & 20 & 15 & 0.60 & $\mathrm{Y}$ \\
\hline & & 124 & 1.6 & 3.3 & 3.0 & 1.9 & 0.33 & \\
\hline \multirow{3}{*}{ NGC 151} & nner-Disk Galaxi & & & & & & & \\
\hline & 75 & 152 & 18 & 23 & 21 & 21 & 0.44 & $\overline{\mathrm{Y}}$ \\
\hline & & 74 & 4.3 & 7.1 & 6.1 & 6.1 & 0.32 & \\
\hline \multirow{2}{*}{ NGC 470} & 155 & 18 & 21 & 30 & 26 & 26 & 0.55 & \\
\hline & & 152 & 2.7 & 7.4 & 11 & 7.4 & 0.46 & \\
\hline \multirow[t]{2}{*}{ NGC 1398} & 96 & 9 & 38 & 46 & 44 & 44 & 0.36 & \\
\hline & & 96 & 2.9 & 8.2 & 7.9 & 7.9 & 0.18 & \\
\hline \multirow[t]{2}{*}{ NGC 2787} & 109 & 160 & 29 & 36 & 36 & 36 & 0.34 & \\
\hline & & 113 & 18 & 22 & 21 & 21 & 0.34 & \\
\hline \multirow[t]{2}{*}{ NGC 2880} & 144 & 82 & 8.0 & 9.0 & 10 & 9.0 & 0.20 & \\
\hline & & 138 & 3.0 & 5.6 & 4.5 & 4.5 & 0.22 & \\
\hline \multirow[t]{2}{*}{ NGC 3266} & 85 & 8 & 10 & 14 & 13 & 13 & 0.29 & $\mathrm{Y}$ \\
\hline & & 90 & 0.8 & 2.4 & 1.8 & 1.8 & 0.12 & \\
\hline \multirow[t]{2}{*}{ NGC 3368} & 172 & 115 & 61 & 80 & 75 & 75 & 0.40 & Y \\
\hline & & 162 & 21 & 35 & 30 & 30 & 0.30 & \\
\hline \multirow[t]{2}{*}{ NGC 3384} & 50 & 132 & 15 & $\ldots$ & 17 & 17 & 0.05 & \\
\hline & & 46 & 2.7 & 15 & 11 & 11 & 0.40 & \\
\hline \multirow[t]{2}{*}{ NGC 3412} & 153 & 100 & 15 & 21 & 21 & 21 & 0.26 & \\
\hline & & 154 & 1.0 & 8.3 & 6.1 & 6.1 & 0.33 & \\
\hline \multirow[t]{2}{*}{ NGC 3945} & 158 & 72 & 32 & 41 & 39 & 39 & 0.29 & $\mathrm{Y}$ \\
\hline & & 158 & 10 & 19 & 18 & 18 & 0.36 & \\
\hline NGC 4143 & 144 & 163 & 17 & $\ldots$ & 28 & 28 & 0.38 & \\
\hline & & 142 & 4.2 & 6.2 & $\ldots$ & 6.2 & 0.25 & \\
\hline
\end{tabular}


Peter Erwin: Double-barred galaxies. I., Online Material p 5

Table 3. continued.

\begin{tabular}{|c|c|c|c|c|c|c|c|c|}
\hline Name & $\begin{array}{r}\text { Outer Disk PA } \\
\circ\end{array}$ & $\begin{array}{r}\text { Bar/Disk PA } \\
\circ\end{array}$ & $\begin{array}{l}a_{\epsilon} \\
\prime \prime\end{array}$ & $a_{\min }$ & $\begin{array}{r}a_{10} \\
\prime \prime\end{array}$ & $\begin{array}{r}L_{\text {bar }} \\
\prime \prime\end{array}$ & $\overline{\epsilon_{\max }}$ & NR? \\
\hline (1) & (2) & (3) & (4) & (5) & (6) & (7) & (8) & (9) \\
\hline \multirow[t]{2}{*}{ NGC 4262} & 153 & 18 & 13 & 20 & 16 & 16 & 0.34 & \\
\hline & & 155 & 3.5 & 4.4 & 4.7 & 4.4 & 0.10 & \\
\hline \multirow[t]{2}{*}{ NGC 4386} & 140 & 134 & 25 & 36 & $\ldots$ & 36 & 0.52 & \\
\hline & & 141 & 2.4 & 3.2 & $\ldots$ & 3.2 & 0.28 & \\
\hline \multirow[t]{2}{*}{ NGC 4612} & 143 & 83 & 17 & 24 & 20 & 20 & 0.22 & \\
\hline & & 144 & 3.0 & 5.7 & 5.5 & 5.5 & 0.21 & \\
\hline \multirow[t]{2}{*}{ NGC 4754} & 23 & 142 & 23 & 27 & 30 & 27 & 0.23 & \\
\hline & & 22 & 6.7 & 9.4 & 12 & 9.4 & 0.23 & \\
\hline \multirow[t]{2}{*}{ NGC 4785} & 81 & 66 & 11 & 13 & 16 & 12 & 0.57 & Y \\
\hline & & 83 & 5.2 & 6.7 & 8.4 & 6.7 & 0.41 & \\
\hline \multirow[t]{2}{*}{ NGC 7007} & 2 & 116 & 6.2 & 8.1 & 7.6 & 7.6 & 0.26 & \\
\hline & & 0 & 2.3 & 3.7 & 2.9 & 2.9 & 0.20 & \\
\hline \multirow[t]{2}{*}{ NGC 7187} & 134 & 66 & 19 & 28 & 22 & 19 & 0.37 & Y \\
\hline & & 134 & 7.2 & 9.4 & 8.6 & 8.6 & 0.12 & \\
\hline \multirow[t]{2}{*}{ UGC 6062} & 25 & 159 & 12 & 17 & 15 & 15 & 0.39 & \\
\hline & & 27 & 1.9 & 2.8 & 3.4 & 2.8 & 0.39 & \\
\hline \multirow[t]{2}{*}{ ESO 378-G020 } & 34 & 81 & 6.9 & 8.3 & 8.8 & 8.3 & 0.37 & \\
\hline & & 39 & 0.8 & 1.3 & . & 1.3 & 0.42 & \\
\hline \multirow[t]{2}{*}{ ESO 443-G017 } & 23 & 160 & 9.6 & 15 & 12 & 12 & 0.48 & \\
\hline & & 31 & 2.3 & 5.5 & 4.2 & 4.2 & 0.28 & \\
\hline
\end{tabular}


Table 4. Deprojected values for bars and inner disks.

\begin{tabular}{|c|c|c|c|c|c|c|c|}
\hline $\begin{array}{l}\text { Name } \\
\text { (1) }\end{array}$ & $\begin{array}{r}\Delta \mathrm{PA} \\
\circ \\
(2)\end{array}$ & $\begin{array}{r}\text { Bar/Disk Size } \\
\text { kpc } \\
(3)\end{array}$ & $\begin{array}{r}\text { Bar/Disk Size } \\
f\left(R_{25}\right) \\
(4)\end{array}$ & $\begin{array}{l}\text { Name } \\
(1)\end{array}$ & $\begin{array}{r}\Delta \mathrm{PA} \\
\circ \\
(2)\end{array}$ & $\begin{array}{r}\text { Bar/Disk Size } \\
\text { kpc } \\
(3)\end{array}$ & $\begin{array}{r}\text { Bar/Disk Size } \\
f\left(R_{25}\right) \\
(4)\end{array}$ \\
\hline \multicolumn{8}{|c|}{ Double-Barred Galaxies } \\
\hline NGC 357 & 67 & $\begin{array}{l}4.01,5.15 \\
0.50,0.71\end{array}$ & $\begin{array}{l}0.36,0.47 \\
0.05,0.06\end{array}$ & NGC 3081 & 62 & $\begin{array}{l}5.02,5.32 \\
0.86,0.87\end{array}$ & $\begin{array}{l}0.55,0.58 \\
0.09,0.10\end{array}$ \\
\hline NGC 718 & 48 & $\begin{array}{l}2.30,3.45 \\
0.18,0.36\end{array}$ & $\begin{array}{l}0.30,0.44 \\
0.02,0.05\end{array}$ & NGC 3275 & 60 & $\begin{array}{l}5.50,7.07 \\
0.50,0.62\end{array}$ & $\begin{array}{l}0.33,0.42 \\
0.03,0.04\end{array}$ \\
\hline NGC 1068 & 28 & $\begin{array}{l}5.02,6.97 \\
1.28,1.45\end{array}$ & $\begin{array}{l}0.33,0.46 \\
0.08,0.10\end{array}$ & NGC 3358 & -49 & $\begin{array}{l}3.13,4.10 \\
0.90,1.52\end{array}$ & $\begin{array}{l}0.17,0.23 \\
0.05,0.09\end{array}$ \\
\hline NGC 1097 & -61 & $\begin{array}{l}6.26,7.62 \\
0.74,0.76\end{array}$ & $\begin{array}{l}0.32,0.39 \\
0.04,0.04\end{array}$ & NGC 3368 & -11 & $\begin{array}{l}4.39,5.40 \\
0.22,0.33\end{array}$ & $\begin{array}{l}0.38,0.47 \\
0.02,0.03\end{array}$ \\
\hline NGC 1241 & -78 & $\begin{array}{l}5.88,5.88 \\
0.49,0.59\end{array}$ & $\begin{array}{l}0.28,0.28 \\
0.02,0.03\end{array}$ & NGC 3393 & -11 & $\begin{array}{l}3.09,3.80 \\
0.46,0.75\end{array}$ & $\begin{array}{l}0.20,0.25 \\
0.03,0.05\end{array}$ \\
\hline NGC 1291 & 24 & $\begin{array}{l}3.41,4.98 \\
0.69,0.92\end{array}$ & $\begin{array}{l}0.30,0.44 \\
0.06,0.08\end{array}$ & NGC 3941 & -64 & $\begin{array}{l}1.39,2.12 \\
0.29,0.41\end{array}$ & $\begin{array}{l}0.23,0.34 \\
0.05,0.07\end{array}$ \\
\hline NGC 1317 & -80 & $\begin{array}{l}4.38,6.19 \\
0.60,0.61\end{array}$ & $\begin{array}{l}0.56,0.80 \\
0.08,0.08\end{array}$ & NGC 3945 & -11 & $\begin{array}{c}4.65,5.67 \\
0.36,0.42\end{array}$ & $\begin{array}{l}0.32,0.38 \\
0.02,0.03\end{array}$ \\
\hline NGC 1433 & 65 & $\begin{array}{l}4.58,5.57 \\
0.33,0.63\end{array}$ & $\begin{array}{l}0.45,0.55 \\
0.03,0.06\end{array}$ & NGC 4303 & -41 & $\begin{array}{l}2.25,2.64 \\
0.15,0.20\end{array}$ & $\begin{array}{l}0.16,0.18 \\
0.01,0.01\end{array}$ \\
\hline NGC 1543 & (66) & $\begin{array}{l}6.50,9.80 \\
0.78,1.08\end{array}$ & $\begin{array}{l}0.46,0.69 \\
0.05,0.08\end{array}$ & NGC 4314 & -10 & $\begin{array}{l}4.29,5.12 \\
0.29,0.36\end{array}$ & $\begin{array}{l}0.59,0.70 \\
0.04,0.05\end{array}$ \\
\hline NGC 1808 & 19 & $\begin{array}{l}4.06,5.78 \\
0.18,0.27\end{array}$ & $\begin{array}{l}0.42,0.60 \\
0.02,0.03\end{array}$ & NGC 4321 & 2 & $\begin{array}{l}4.32,4.55 \\
0.64,0.78\end{array}$ & $\begin{array}{l}0.26,0.28 \\
0.04,0.05\end{array}$ \\
\hline NGC 2217 & -24 & $\begin{array}{l}3.54,4.79 \\
0.77,1.08\end{array}$ & $\begin{array}{l}0.29,0.39 \\
0.06,0.09\end{array}$ & NGC 4340 & 4 & $\begin{array}{l}4.24,5.22 \\
0.38,0.50\end{array}$ & $\begin{array}{l}0.54,0.67 \\
0.05,0.06\end{array}$ \\
\hline NGC 2642 & -30 & $\begin{array}{l}7.01,7.29 \\
0.41,0.55\end{array}$ & $\begin{array}{l}0.42,0.43 \\
0.02,0.03\end{array}$ & NGC 4503 & 69 & $\begin{array}{l}1.74,2.05 \\
0.32,0.53\end{array}$ & $\begin{array}{l}0.22,0.26 \\
0.04,0.07\end{array}$ \\
\hline NGC 2646 & (74) & $\begin{array}{l}4.33,5.69 \\
0.56,0.89\end{array}$ & $\begin{array}{l}0.43,0.56 \\
0.06,0.09\end{array}$ & NGC 4725 & -84 & $\begin{array}{l}7.18,7.61 \\
0.45,0.55\end{array}$ & $\begin{array}{l}0.37,0.39 \\
0.02,0.03\end{array}$ \\
\hline NGC 2681 & & $\begin{array}{l}4.17,5.00 \\
1.50,1.58 \\
0.14,0.28\end{array}$ & $\begin{array}{l}0.46,0.55 \\
0.17,0.17 \\
0.02,0.03\end{array}$ & NGC 4736 & 60 & $\begin{array}{l}3.27,4.44 \\
0.34,0.62\end{array}$ & $\begin{array}{l}0.38,0.52 \\
0.04,0.07\end{array}$ \\
\hline NGC 2859 & 80 & $\begin{array}{l}4.38,5.54 \\
0.49,0.75\end{array}$ & $\begin{array}{l}0.29,0.37 \\
0.03,0.05\end{array}$ & NGC 4785 & 81 & $\begin{array}{l}2.80,3.06 \\
0.36,0.44\end{array}$ & $\begin{array}{l}0.21,0.23 \\
0.03,0.03\end{array}$ \\
\hline NGC 2950 & 80 & $\begin{array}{l}2.16,2.79 \\
0.27,0.33\end{array}$ & $\begin{array}{l}0.37,0.48 \\
0.05,0.06\end{array}$ & NGC 4984 & $(25)$ & $\begin{array}{l}2.94,3.14 \\
0.36,0.44\end{array}$ & $\begin{array}{l}0.46,0.49 \\
0.06,0.07\end{array}$ \\
\hline NGC 2962 & 60 & $\begin{array}{l}4.71,6.98 \\
0.84,1.01\end{array}$ & $\begin{array}{l}0.41,0.61 \\
0.07,0.09\end{array}$ & NGC 5365 & -57 & $\begin{array}{l}5.43,6.74 \\
0.63,0.90\end{array}$ & $\begin{array}{l}0.41,0.51 \\
0.05,0.07\end{array}$ \\
\hline
\end{tabular}

Deprojected relative position angles and sizes for double-barred and inner-disk galaxies. For each galaxy, the first line contains values for the outer bar and the second line contains values for the inner bar (double-barred galaxies) or inner disk. Column (1): galaxy name. Column (2): difference in position angle between inner bar or disk and outer bar. Positive $=$ inner bar/disk leads outer bar, negative $=$ inner bar/disk trails, based on the sense of rotation (Table 1 or 2). Values in parentheses are for galaxies where the sense of rotation is undefined. No values are given for NGC 2681 because there are, in effect, two "outer" bars. Column (3) bar/disk linear sizes, in kpc. The first number is $a_{\epsilon}$, the second is $L_{\mathrm{bar}}$. Column (4) bar/disk sizes relative to galaxy size $R_{25}$; the first number is $a_{\epsilon} / R_{25}$, the second is $L_{\mathrm{bar}} / R_{25}$. Note that NGC 3368, 3945, 4785, and 7187 have both inner bars and inner disks, and are thus included in each section. 
Table 4. continued.

\begin{tabular}{|c|c|c|c|c|c|c|c|}
\hline $\begin{array}{l}\text { Name } \\
\text { (1) } \\
\end{array}$ & $\begin{array}{r}\mathrm{PA} \\
\circ \\
(2) \\
\end{array}$ & $\begin{array}{r}\text { Bar/Disk Size } \\
\text { kpc } \\
(3) \\
\end{array}$ & $\begin{array}{r}\text { Bar/Disk Size } \\
f\left(R_{25}\right) \\
(4) \\
\end{array}$ & $\begin{array}{l}\text { Name } \\
(1) \\
\end{array}$ & $\begin{array}{r}\mathrm{PA} \\
\circ \\
(2) \\
\end{array}$ & $\begin{array}{r}\text { Bar/Disk Size } \\
\text { kpc } \\
(3) \\
\end{array}$ & $\begin{array}{r}\text { Bar/Disk Size } \\
f\left(R_{25}\right) \\
(4) \\
\end{array}$ \\
\hline NGC 5728 & -47 & $\begin{array}{r}10.91,13.84 \\
0.42,0.86\end{array}$ & $\begin{array}{l}0.66,0.83 \\
0.03,0.05\end{array}$ & NGC 7716 & 37 & $\begin{array}{l}3.66,4.16 \\
0.53,0.92\end{array}$ & $\begin{array}{l}0.35,0.39 \\
0.05,0.09\end{array}$ \\
\hline NGC 5850 & -59 & $\begin{array}{r}11.67,13.89 \\
1.14,1.37\end{array}$ & $\begin{array}{l}0.53,0.64 \\
0.05,0.06\end{array}$ & Mrk 573 & -83 & $\begin{array}{l}2.98,3.97 \\
0.41,0.71\end{array}$ & $\begin{array}{l}0.23,0.30 \\
0.03,0.05\end{array}$ \\
\hline NGC 6654 & 77 & $\begin{array}{l}3.71,5.42 \\
0.45,0.70\end{array}$ & $\begin{array}{l}0.34,0.50 \\
0.04,0.06\end{array}$ & UGC 524 & 24 & $\begin{array}{l}6.04,7.82 \\
0.73,0.80\end{array}$ & $\begin{array}{l}0.28,0.36 \\
0.03,0.04\end{array}$ \\
\hline NGC 6684 & $(60)$ & $\begin{array}{l}2.64,2.84 \\
0.23,0.33\end{array}$ & $\begin{array}{l}0.33,0.35 \\
0.03,0.04\end{array}$ & ESO 215-G031 & 7 & $\begin{array}{l}6.40,7.92 \\
0.92,1.07\end{array}$ & $\begin{array}{l}0.56,0.69 \\
0.08,0.09\end{array}$ \\
\hline NGC 6782 & -26 & $\begin{array}{l}6.97,8.36 \\
1.05,1.17\end{array}$ & $\begin{array}{l}0.45,0.54 \\
0.07,0.08\end{array}$ & ESO 320-G030 & 56 & $\begin{array}{l}4.99,8.02 \\
0.80,0.92\end{array}$ & $\begin{array}{l}0.38,0.62 \\
0.06,0.07\end{array}$ \\
\hline NGC 7098 & -31 & $\begin{array}{l}5.63,7.85 \\
1.24,1.63\end{array}$ & $\begin{array}{l}0.34,0.47 \\
0.07,0.10\end{array}$ & ESO 443-G039 & (23) & $\begin{array}{l}2.22,3.83 \\
0.44,0.58\end{array}$ & $\begin{array}{l}0.29,0.50 \\
0.06,0.08\end{array}$ \\
\hline NGC 7187 & -10 & $\begin{array}{l}3.42,3.42 \\
0.45,0.64\end{array}$ & $\begin{array}{l}0.51,0.51 \\
0.07,0.09\end{array}$ & ESO 447-G030 & -34 & $\begin{array}{l}3.67,3.95 \\
0.63,0.81\end{array}$ & $\begin{array}{l}0.47,0.50 \\
0.08,0.10\end{array}$ \\
\hline NGC 7280 & 78 & $\begin{array}{l}1.36,3.34 \\
0.18,0.24\end{array}$ & $\begin{array}{l}0.18,0.43 \\
0.02,0.03\end{array}$ & IRAS $03565+2139$ & 60 & $\begin{array}{l}6.37,7.35 \\
0.82,0.97\end{array}$ & $\begin{array}{l}0.77,0.88 \\
0.10,0.12\end{array}$ \\
\hline \multicolumn{8}{|c|}{ Inner-Disk Galaxies } \\
\hline NGC 151 & -86 & $\begin{array}{r}9.89,11.53 \\
1.02,1.45\end{array}$ & $\begin{array}{l}0.38,0.44 \\
0.04,0.05\end{array}$ & NGC 4262 & $(46)$ & $\begin{array}{l}1.04,1.27 \\
0.26,0.33\end{array}$ & $\begin{array}{l}0.25,0.31 \\
0.06,0.08\end{array}$ \\
\hline NGC 470 & -64 & $\begin{array}{l}4.53,5.61 \\
0.41,1.13\end{array}$ & $\begin{array}{l}0.36,0.44 \\
0.03,0.09\end{array}$ & NGC 4386 & $(10)$ & $\begin{array}{l}3.29,4.74 \\
0.31,0.42\end{array}$ & $\begin{array}{l}0.34,0.49 \\
0.03,0.04\end{array}$ \\
\hline NGC 1398 & 87 & $\begin{array}{l}4.19,4.85 \\
0.23,0.62\end{array}$ & $\begin{array}{l}0.25,0.29 \\
0.01,0.04\end{array}$ & NGC 4612 & $(68)$ & $\begin{array}{l}1.64,1.93 \\
0.22,0.41\end{array}$ & $\begin{array}{l}0.30,0.35 \\
0.04,0.07\end{array}$ \\
\hline NGC 2787 & -58 & $\begin{array}{l}1.58,1.96 \\
0.66,0.77\end{array}$ & $\begin{array}{l}0.46,0.57 \\
0.19,0.22\end{array}$ & NGC 4754 & (73) & $\begin{array}{l}3.72,4.37 \\
0.55,0.77\end{array}$ & $\begin{array}{l}0.33,0.39 \\
0.05,0.07\end{array}$ \\
\hline NGC 2880 & -62 & $\begin{array}{l}1.28,1.44 \\
0.32,0.48\end{array}$ & $\begin{array}{l}0.19,0.22 \\
0.05,0.07\end{array}$ & NGC 4785 & -35 & $\begin{array}{l}2.80,3.06 \\
1.17,1.51\end{array}$ & $\begin{array}{l}0.21,0.23 \\
0.09,0.11\end{array}$ \\
\hline NGC 3266 & $(85)$ & $\begin{array}{l}1.47,1.92 \\
0.10,0.22\end{array}$ & $\begin{array}{l}0.26,0.34 \\
0.02,0.04\end{array}$ & NGC 7007 & $(72)$ & $\begin{array}{l}1.86,2.28 \\
0.42,0.53\end{array}$ & $\begin{array}{l}0.18,0.22 \\
0.04,0.05\end{array}$ \\
\hline NGC 3368 & -52 & $\begin{array}{l}4.39,5.40 \\
1.09,1.56\end{array}$ & $\begin{array}{l}0.38,0.47 \\
0.09,0.13\end{array}$ & NGC 7187 & 70 & $\begin{array}{l}3.42,3.42 \\
1.18,1.41\end{array}$ & $\begin{array}{l}0.51,0.51 \\
0.18,0.21\end{array}$ \\
\hline NGC 3384 & $(85)$ & $\begin{array}{l}1.73,1.96 \\
0.15,0.62\end{array}$ & $\begin{array}{l}0.19,0.21 \\
0.02,0.07\end{array}$ & UGC 6062 & (64) & $\begin{array}{l}2.98,3.72 \\
0.33,0.49\end{array}$ & $\begin{array}{l}0.48,0.60 \\
0.05,0.08\end{array}$ \\
\hline NGC 3412 & (66) & $\begin{array}{l}1.18,1.65 \\
0.05,0.33\end{array}$ & $\begin{array}{l}0.20,0.28 \\
0.01,0.06\end{array}$ & ESO 378-G020 & (53) & $\begin{array}{l}1.93,2.32 \\
0.15,0.24\end{array}$ & $\begin{array}{l}0.27,0.32 \\
0.02,0.03\end{array}$ \\
\hline NGC 3945 & -87 & $\begin{array}{l}4.65,5.67 \\
0.94,1.68\end{array}$ & $\begin{array}{l}0.32,0.38 \\
0.06,0.11\end{array}$ & ESO 443-G017 & -67 & $\begin{array}{l}2.36,2.95 \\
0.44,0.81\end{array}$ & $\begin{array}{l}0.29,0.37 \\
0.06,0.10\end{array}$ \\
\hline NGC 4143 & 37 & $\begin{array}{l}1.49,2.45 \\
0.32,0.48\end{array}$ & $\begin{array}{l}0.28,0.46 \\
0.06,0.09\end{array}$ & & & & \\
\hline
\end{tabular}

